\title{
Sustaining mechanism of Taylor-Görtler-like vortices in a streamwise-rotating channel flow
}

\author{
Zixuan Yang $\odot,{ }^{1}$ Bing-Qing Deng $\odot,{ }^{2,}{ }^{*}$ Bing-Chen Wang, ${ }^{3}$ and Lian Shen $\oplus^{2}$ \\ ${ }^{1}$ State Key Laboratory of Nonlinear Mechanics, Institute of Mechanics, \\ Chinese Academy of Sciences, Beijing 100190, China \\ ${ }^{2}$ Department of Mechanical Engineering \& St. Anthony Falls Laboratory, \\ University of Minnesota, Minneapolis, Minnesota 55455, USA \\ ${ }^{3}$ Department of Mechanical Engineering, University of Manitoba, Winnipeg, Manitoba R3T 5V6, Canada
}

(Received 24 October 2019; accepted 20 March 2020; published 21 April 2020)

\begin{abstract}
Energy transport in the spectral space is analyzed to study the mechanism underlying the Taylor-Görtler-like (TGL) vortices that appear as two layers of streamwise-elongated roll cells in a turbulent channel flow subjected to fast streamwise system rotation. The transport equation of the velocity-spectrum tensor in a rotating frame is derived to study the budget balance of energy spectra at different length scales. Two new terms, namely, the rotation-induced redistribution term and rotation-induced wall-normal diffusion term, are defined to reflect the effect of the imposed system rotation on the energy transport process. By analyzing the data obtained from direct numerical simulation, it is discovered that four key processes are responsible for sustaining the motion of the TGL vortices. The first process corresponds to the energy production at the characteristic length scales of the TGL vortices that drains energy from the mean flow to the TGL vortices. The second process is the rotation-induced energy redistribution from the streamwise velocity fluctuations to the wall-normal and spanwise velocity fluctuations that form the vortex structures on a cross-stream plane. The third process is the energy diffusion from the near-wall region to the channel center, which is enhanced due to the occurrence of the TGL vortices and, in turn, feeds energy to the vortices. The last process is the inverse interscale energy transfer, through which the large-scale TGL vortices absorb energy from small-scale eddies.
\end{abstract}

DOI: 10.1103/PhysRevFluids.5.044601

\section{INTRODUCTION}

System rotations can induce large-scale Taylor-Görtler-like (TGL) vortex structures in turbulent channel flows [1,2]. The occurrence of the TGL vortices further alters the turbulence field through a global transport of momentum and energy across the channel. A thorough investigation on the TGL vortices is crucial for understanding the dynamics of turbulent flows in rotating channels.

In the pressure-driven turbulent channel flows and turbulent Couette flows subjected to spanwise system rotation [3-9], the TGL vortices appear as one layer of large-scale streamwise-elongated roll cells. The TGL vortices are essentially induced by the inviscid local linear instability [10-14]. The TGL vortices in the spanwise-rotating channel flow occur at a low critical Reynolds number [13], forming a streamwise-independent and spanwise-periodic pattern. The readers are referred to the recent study of the Reynolds stress transport in the spanwise-rotating channel flow by Kawata and Alfredsson [9] and the references therein for more details of the instability analyses of the spanwise-rotating channel flow.

\footnotetext{
*Corresponding author: bdeng@umn.edu
} 
TABLE I. Coriolis terms in the transport equations of Reynolds stress components $\left\langle u_{i}^{\prime} u_{j}^{\prime}\right\rangle$ in the spanwiserotating and streamwise-rotating channel flows.

\begin{tabular}{lcc}
\hline \hline Component & Spanwise-rotating channel flow & Streamwise-rotating channel flow \\
\hline$\left\langle u_{1}^{\prime} u_{1}^{\prime}\right\rangle$ & $4 \Omega\left\langle u_{1}^{\prime} u_{2}^{\prime}\right\rangle$ & 0 \\
$\left\langle u_{2}^{\prime} u_{2}^{\prime}\right\rangle$ & $-4 \Omega\left\langle u_{1}^{\prime} u_{2}^{\prime}\right\rangle$ & $4 \Omega\left\langle u_{2}^{\prime} u_{3}^{\prime}\right\rangle$ \\
$\left\langle u_{3}^{\prime} u_{3}^{\prime}\right\rangle$ & 0 & $-4 \Omega\left\langle u_{2}^{\prime} u_{3}^{\prime}\right\rangle$ \\
$\left\langle u_{1}^{\prime} u_{2}^{\prime}\right\rangle$ & $-2 \Omega\left(\left\langle u_{1}^{\prime} u_{1}^{\prime}\right\rangle-\left\langle u_{2}^{\prime} u_{2}^{\prime}\right\rangle\right)$ & $2 \Omega\left\langle u_{1}^{\prime} u_{3}^{\prime}\right\rangle$ \\
$\left\langle u_{1}^{\prime} u_{3}^{\prime}\right\rangle$ & 0 & $-2 \Omega\left\langle u_{1}^{\prime} u_{2}^{\prime}\right\rangle$ \\
$\left\langle u_{2}^{\prime} u_{3}^{\prime}\right\rangle$ & 0 & $-2 \Omega\left(\left\langle u_{2}^{\prime} u_{2}^{\prime}\right\rangle-\left\langle u_{3}^{\prime} u_{3}^{\prime}\right\rangle\right)$ \\
\hline \hline
\end{tabular}

On the subject of the present research of streamwise-rotating channel flows, only a few studies focusing on the TGL vortices have been conducted. By analyzing their direct numerical simulation (DNS) data, Yang and Wang [15] observed a two-layer pattern of the TGL vortices in a streamwise-rotating channel flow. They reported that, as the rotation number increases, the streamwise characteristic length scales of the TGL vortices increase monotonically, but their spanwise characteristic length scales remain almost unchanged. Similar to the one-layer roll cells in the spanwise-rotating channel flow, the two-layer pattern of TGL vortices in the streamwise-rotating channel flow also occur at a low Reynolds number $(\mathrm{Re}=70$ based on the velocity at the channel center and one-half the channel height) [2]. Yang et al. [16] further investigated the effects of streamwise system rotation on the pressure fluctuations. They discovered that the occurrence of the TGL vortices significantly enhances pressure fluctuations at a very high rotation number. Dai et al. [17] studied the flow structures under the effects of the TGL vortices and mean spanwise motion in the streamwise-rotating channel flow $[18,19]$. The above studies of the TGL vortices in streamwise-rotating turbulent channel flows mainly focused on the kinematic properties, in terms of the characteristic length scales of TGL vortices and their influence on the mean and secondary flow fields. However, the dynamics of the TGL vortices have not been investigated; specifically, the physical processes associated with the production, dissipation, and diffusion of turbulent kinetic energy (TKE) for sustaining the motion of the TGL vortices are unclear, and the effect of the TGL vortices on the energy transport has not been studied systematically.

Insights into flow physics, coherent structures, and energy balance can be sought by investigating the transport equation of Reynolds stresses. Following the pioneering work of Mansour et al. [20], the budget balance of Reynolds stresses has been studied extensively using DNS in the context of turbulent channel flows [21-23], turbulent Couette flows [24,25], turbulent boundary-layer flows [24,26], and turbulent channel flows subjected to spanwise system rotation [3-5,9,12,27] and streamwise system rotation [15]. In the rotating channel flows, additional Coriolis terms enter the transport equations of Reynolds stresses, and play important roles in the balances of TKE and momentum fluxes. Table I compares the Coriolis terms in the transport equations of Reynolds stresses between the spanwise- and streamwise-rotating channel flows. In the table, $x_{i}$ and $u_{i}$ denote the coordinates and velocity components, respectively, subscripts 1-3 denote the variables corresponding to the streamwise, wall-normal, and spanwise directions of the flow, respectively, $\Omega$ represents the norm of the angular velocity of the system rotation, and a pair of angular brackets are used to denote averaging over time and homogeneous directions, i.e., the $x_{1}$ and $x_{3}$ directions. It is seen from the table that, in the spanwise-rotating channel flow, the Coriolis terms lead to an energy redistribution between $\left\langle u_{1}^{\prime} u_{1}^{\prime}\right\rangle$ and $\left\langle u_{2}^{\prime} u_{2}^{\prime}\right\rangle$ of which the magnitude is proportional to the shear component $\left\langle u_{1}^{\prime} u_{2}^{\prime}\right\rangle$. Meanwhile, the Coriolis term imposes an additional production effect on the budget balance of $\left\langle u_{1}^{\prime} u_{2}^{\prime}\right\rangle$, which tends to cause enhancement and suppression of the turbulence intensity on the pressure and suction sides of the channel, respectively. The Coriolis terms of $\left\langle u_{3}^{\prime} u_{3}^{\prime}\right\rangle,\left\langle u_{1}^{\prime} u_{3}^{\prime}\right\rangle$, and $\left\langle u_{2}^{\prime} u_{3}^{\prime}\right\rangle$ are zero in the spanwise-rotating channel flow. Compared to the spanwise system rotation, the effects of the streamwise system rotation on the budget balances of Reynolds stresses show the following 
three major differences. First, in the streamwise-rotating channel flow, the Coriolis term is absent in the transport equation of $\left\langle u_{1}^{\prime} u_{1}^{\prime}\right\rangle$. It was discovered in our previous studies $[15,28]$ that the streamwise system rotation influenced the budget balance of $\left\langle u_{1}^{\prime} u_{1}^{\prime}\right\rangle$ mainly through the pressure-redistribution effect, which caused additional TKE transport from $\left\langle u_{1}^{\prime} u_{1}^{\prime}\right\rangle$ to $\left\langle u_{2}^{\prime} u_{2}^{\prime}\right\rangle$ and $\left\langle u_{3}^{\prime} u_{3}^{\prime}\right\rangle$ in the streamwiserotating channel flow, leading to the suppression of $\left\langle u_{1}^{\prime} u_{1}^{\prime}\right\rangle$ at high rotation numbers. Second, the Coriolis terms in the transport equations of $\left\langle u_{1}^{\prime} u_{3}^{\prime}\right\rangle$ and $\left\langle u_{2}^{\prime} u_{3}^{\prime}\right\rangle$ are nonzero in the streamwise-rotating channel flow. This leads to the nonzero behavior of $\left\langle u_{1}^{\prime} u_{3}^{\prime}\right\rangle$ and $\left\langle u_{2}^{\prime} u_{3}^{\prime}\right\rangle$ in the streamwise-rotating channel flow. The $\left\langle u_{2}^{\prime} u_{3}^{\prime}\right\rangle$ component further appears in the Coriolis terms in the transport equations of $\left\langle u_{2}^{\prime} u_{2}^{\prime}\right\rangle$ and $\left\langle u_{3}^{\prime} u_{3}^{\prime}\right\rangle$ to redistribute energy between them. Finally, although the Coriolis term in the transport equation of $\left\langle u_{1}^{\prime} u_{2}^{\prime}\right\rangle$ is nonzero in the streamwise-rotating channel flow, its influence on $\left\langle u_{1}^{\prime} u_{2}^{\prime}\right\rangle$ is not significant as in the spanwise-rotating channel flow. As explained in detail in our previous study [15], the wall-normal distribution of $\left\langle u_{1}^{\prime} u_{2}^{\prime}\right\rangle$ remains stable in the streamwise-rotating channel flows at various rotation numbers because it is the dominant component of the total shear stress (which is independent of the rotation number) in the central region of the channel.

The above studies of the budget balance of Reynolds stresses were conducted in the physical space. Alternatively, the energy transport processes can be investigated in the spectral space, which is advantageous in identifying the characteristic length scales of the physical processes and quantifying the TKE transport across the cascade of wavelengths corresponding to the turbulence structures. To this purpose, Lumley [29] derived the spectral energy equation to study the scale-by-scale energy transport in the context of inhomogeneous turbulent flows. Since the early work of Lumley [29], the transport equation of the velocity-spectrum tensor has been analyzed in wall-bounded turbulence to study the dynamics of coherent structures. Mizuno [30] performed DNS to study the interscale and wall-normal transport of energy in turbulent channel flows through the analyses of the energy spectra. They found that the dominant structures at different scales in the energy transport process are self-similar. Lee and Moser [31,32] further studied the effect of the Reynolds number on the transport equations of the four nontrivial components of the velocity-spectrum tensor. They pointed out that streamwise-elongated structures are important in the energy transport in a turbulent channel flow. Kawata and Alfredsson [33] and Kawata and Tsukahara [34] studied the transport equation of energy spectra in a turbulent Couette flow. They observed that the inverse interscale energy transport process was crucial for sustaining the motion of large-scale vortex structures. These studies [30-34] are fairly recent and have focused on channel flows in a stationary frame. They indicate that the transport equation of energy spectra is a useful tool for analyzing the flow physics, which is employed in the present paper to investigate the TGL vortices in the streamwise-rotating turbulent channel flow.

The main objective of the present research is to study the sustaining mechanism of the TGL vortices in a streamwise-rotating channel flow. The dominant physical processes that provide energy to sustain the motions of the TGL vortices are investigated through the analyses of the transport equation of energy spectra. The remainder of this paper is organized as follows: In Sec. II, the cases and numerical method for generating the DNS database are introduced; in Sec. III, the effects of the TGL vortices on the energy spectra are demonstrated; in Sec. IV, the transport equation of energy spectra is analyzed; and in Sec. V, the main findings of this research are summarized.

\section{DNS DATA OF STREAMWISE-ROTATING TURBULENT CHANNEL FLOW}

The present study of the sustaining mechanism of the TGL vortices in a streamwise-rotating channel flow is conducted based on the analyses of the DNS database recently obtained by Yang and Wang [15]. This database includes six rotation numbers ranging from $\operatorname{Ro}_{\tau}=2 \Omega h / u_{\tau}=0$ to 150 , where $\Omega, h$, and $u_{\tau}$ represent the angular speed of the system rotation, one-half the channel height, and the mean friction velocity of the two channel walls, respectively. It was discovered in our previous study [15] that the characteristic streamwise length scales and the intensities of the TGL vortices both increase monotonically as the rotation number increases. To focus on the mechanism sustaining the TGL vortices, the case for the highest rotation number $\operatorname{Ro}_{\tau}=150$ is 
TABLE II. Summary of DNS cases and parameters.

\begin{tabular}{lcc}
\hline \hline $\mathrm{Ro}_{\tau}$ & $L_{1} \times L_{2} \times L_{3}$ & \multicolumn{1}{c}{$N_{1} \times N_{2} \times N_{3}$} \\
\hline 0 & $16 \pi h \times 2 h \times 8 \pi h$ & $512 \times 128 \times 512$ \\
150 & $512 \pi h \times 2 h \times 8 \pi h$ & $16384 \times 128 \times 512$ \\
\hline \hline
\end{tabular}

chosen as a representation of the streamwise-rotating channel flows because, in this case, the TGL vortices are the most intensive, and their streamwise length scales are the largest among all rotation numbers tested [15]. To identify the effects of imposed streamwise system rotation on the energy transport processes, a nonrotating channel flow for $\mathrm{Ro}_{\tau}=0$ is analyzed as a comparative case. Table II summarizes the key parameters of these two DNS cases, including the rotation number $\operatorname{Ro}_{\tau}$, domain size $L_{1} \times L_{2} \times L_{3}$, and number of grid points $N_{1} \times N_{2} \times N_{3}$. The computational domain size was chosen based on a systematic study of the effect of the domain size on the simulation results [15]. The Reynolds number is fixed at $\operatorname{Re}_{\tau}=u_{\tau} h / v=180$, where $v$ is the kinematic viscosity.

The DNS was conducted by solving the following continuity and momentum equations for an incompressible flow subjected to a system rotation:

$$
\begin{gathered}
\frac{\partial u_{i}}{\partial x_{i}}=0, \\
\frac{\partial u_{i}}{\partial t}+u_{k} \frac{\partial u_{i}}{\partial x_{k}}=-\frac{1}{\rho} \frac{\partial p}{\partial x_{i}}+v \frac{\partial^{2} u_{i}}{\partial x_{k} \partial x_{k}}-2 \varepsilon_{i j k} \Omega_{j} u_{k}-\frac{\Pi}{\rho} \delta_{i 1} .
\end{gathered}
$$

Here, $\rho$ represents the density of the fluid, $p$ is the pressure, $\Omega_{j}$ is the angular velocity of the system rotation in the $j$ th direction, $\varepsilon_{i j k}$ is the Levi-Civita symbol, $\Pi$ is the constant mean streamwise pressure gradient that drives the flow, and $\delta_{i j}$ is the Kronecker $\delta$. For a streamwise-rotating channel flow, $\boldsymbol{\Omega}=[\Omega, 0,0]^{T}$. Periodic boundary conditions are imposed in the streamwise and spanwise directions, and the no-slip boundary condition is prescribed at two solid walls at $x_{2}= \pm h$. Equations (1) and (2) are solved using an in-house pseudospectral method code. This computer code has been extensively tested in the context of nonrotating [35], streamwise-rotating [15,16], and spanwise-rotating [36,37] turbulent channel flows.

\section{EFFECT OF TGL VORTICES ON VELOCITY FLUCTUATIONS}

Before starting the analyses of the sustaining mechanism of the TGL vortices, we first show their major features through the illustration of the instantaneous flow field and energy spectra. Figure 1 visualizes the TGL vortices using the vectors consisting of instantaneous velocity fluctuations

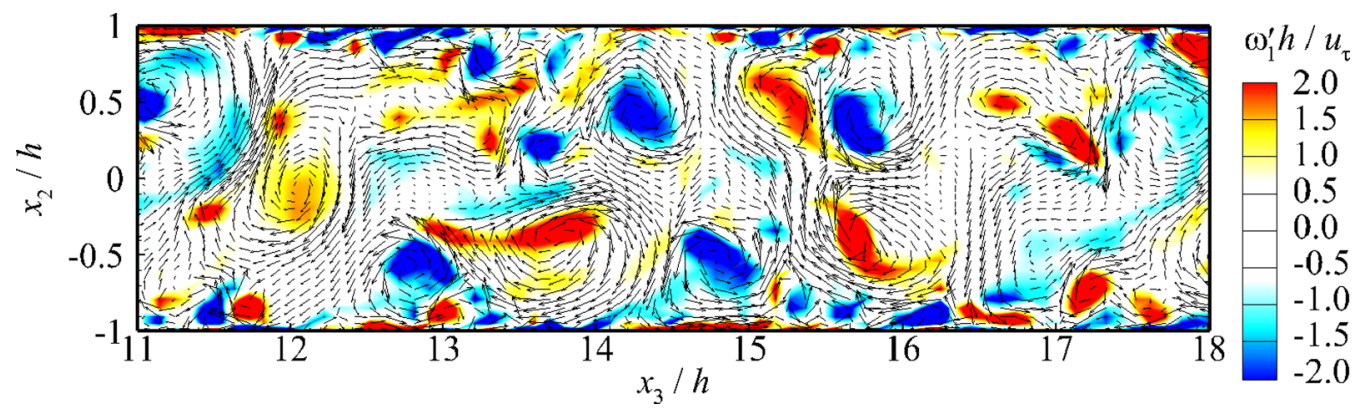

FIG. 1. Visualization of TGL vortices using vectors consisting of instantaneous velocity fluctuations $u_{2}^{\prime}$ and $u_{3}^{\prime}$ and contours of instantaneous streamwise vorticity fluctuations $\omega_{1}^{\prime}$ on an $x_{3}-x_{2}$ plane of the streamwiserotating channel flow at $\mathrm{Ro}_{\tau}=150$. 

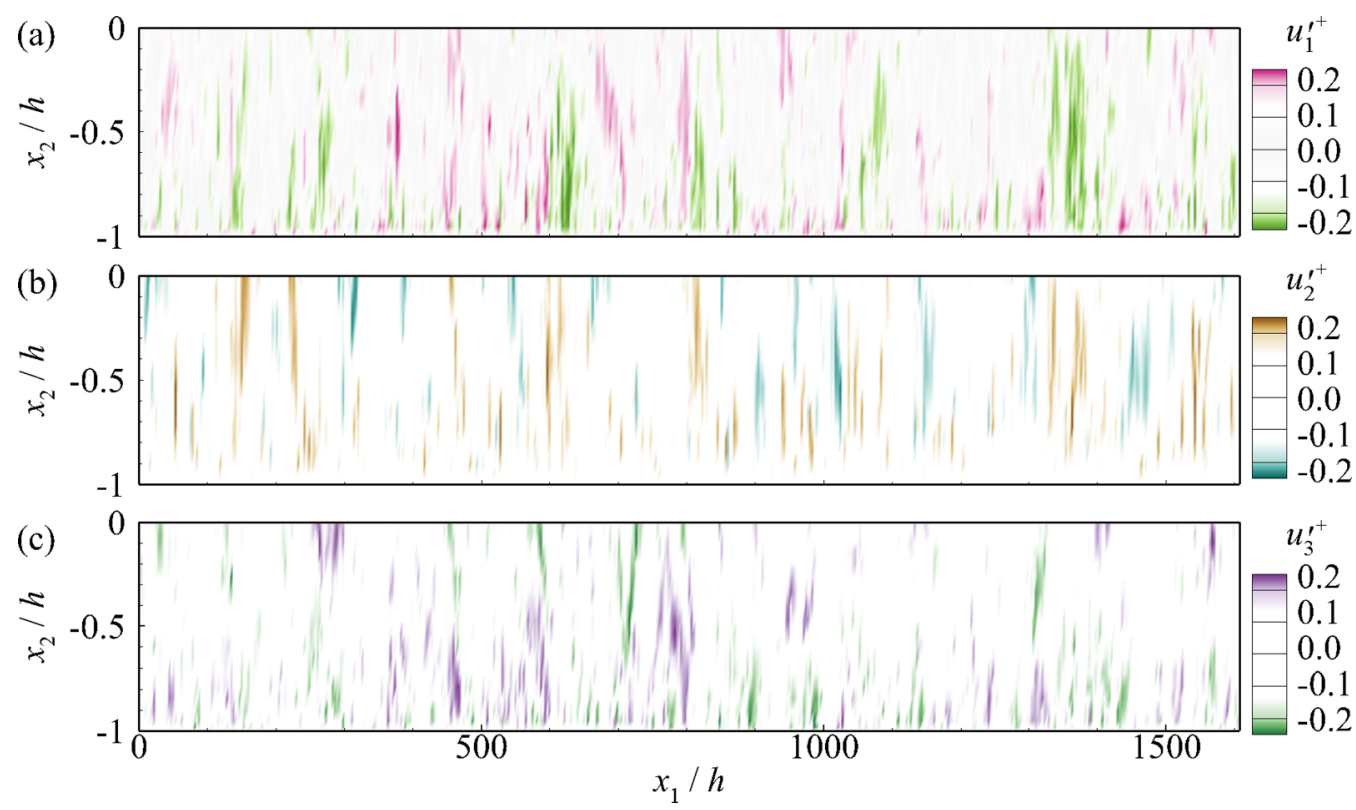

FIG. 2. Contours of instantaneous velocity fluctuations (a) $u_{1}^{\prime+}$, (b) $u_{2}^{\prime+}$, and (c) $u_{3}^{\prime+}$ on an $x_{1}-x_{2}$ plane of the streamwise-rotating channel flow at $\mathrm{Ro}_{\tau}=150$. Note that the ratio between the scales in the $x_{1}$ and $x_{2}$ directions has a large value of 300:1.

$u_{2}^{\prime}$ and $u_{3}^{\prime}$ and contours of the instantaneous streamwise vorticity fluctuations $\omega_{1}^{\prime}$ on an $x_{3}-x_{2}$ plane. Because the spanwise domain size is much larger than the channel height, only a portion of the spanwise domain (from $x_{3} / h=11$ to 18 ) is plotted to show the vortex structures more clearly. It is seen from Fig. 1 that the large-scale TGL vortex structures form a two-layer pattern for $13 \leqslant x_{3} / h \leqslant 18$. This is consistent with the TGL vortices observed by Yang and Wang [15] based on the time-averaged velocity fluctuations. These two-layer vortex structures induce large magnitude wall-normal velocity fluctuations around $x_{2} / h= \pm 0.5$ and large magnitude spanwise velocity fluctuations near the walls around $x_{2} / h= \pm 0.9$ and around the channel center $x_{2} / h=0$. At some other spanwise locations, such as $x_{3} / h=12$, one-layer vortices occur due to the coalescence of corotating vortices, which enhance the wall-normal velocity fluctuations around the channel center $x_{2} / h=0$. The instantaneous TGL vortices shown in Fig. 1 indicate that the occurrence of the TGL vortices in the streamwise-rotating turbulent channel has a significant impact on the secondary flow pattern of the turbulence in the cross-stream directions, which leads to the enhancement of the wall-normal and spanwise velocity fluctuations.

The above results of instantaneous vortex structures show the distribution of the TGL vortices in the wall-normal and spanwise directions. To further demonstrate the streamwise distribution of the TGL vortices, the contours of $u_{i}^{\prime+}$ are depicted in Fig. 2 where the superscript " + " denotes physical quantities nondimensionalized using $u_{\tau}$ and $v / u_{\tau}$ as the characteristic velocity and length scales, respectively, with $u_{\tau}=\sqrt{v d\left\langle u_{1}\right\rangle /\left.d x_{2}\right|_{x_{2}=-h}}$ being the streamwise wall friction velocity. Note that the ratio between the scales in the $x_{1}$ and $x_{2}$ directions is set to a large value of 300:1 in the figure to show the entire streamwise computational domain. Therefore, the streamwise extent of each vortex structure is actually much larger than its wall-normal size. From Figs. 2(a) and 2(c), it is seen that the streamwise and spanwise velocity fluctuations, i.e., $u_{1}^{\prime+}$ and $u_{3}^{\prime+}$, are more active in the near-wall region around $x_{2} / h=-0.9$ than in the center region of the channel around $x_{2} / h=0$. In contrast, as shown in Fig. 2(b), the wall-normal velocity fluctuations $u_{2}^{\prime+}$ are more active around $x_{2} / h=-0.5$ where the cores of one of the two layers of the TGL vortices are concentrated (Fig. 1). 

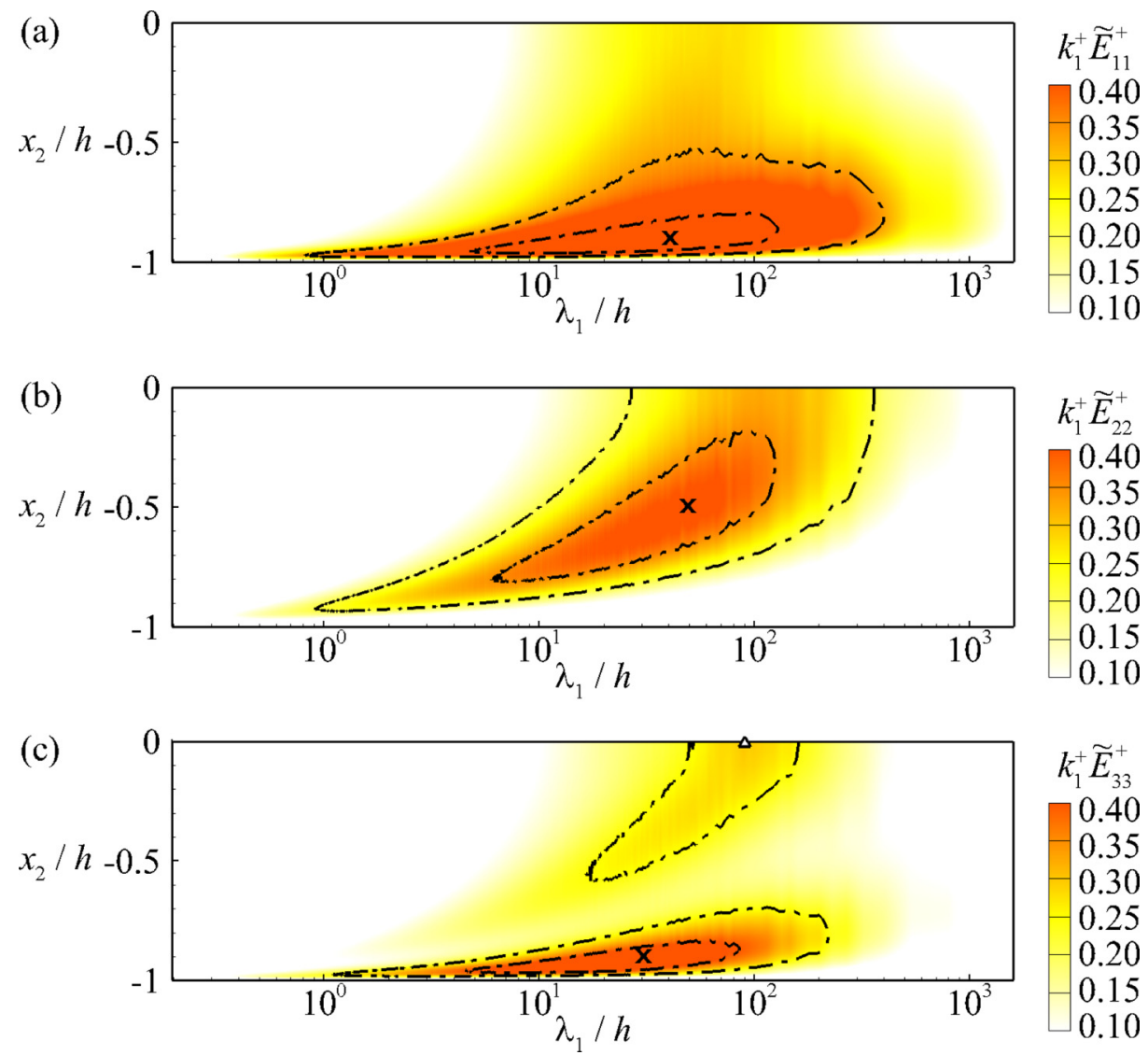

FIG. 3. Contours of premultiplied energy spectra (a) $k_{1}^{+} \tilde{E}_{11}^{+}$, (b) $k_{1}^{+} \tilde{E}_{22}^{+}$, and (c) $k_{1}^{+} \tilde{E}_{33}^{+}$on a $\lambda_{1}-x_{2}$ plane in the streamwise-rotating channel flow at $\operatorname{Ro}_{\tau}=150$. The cross symbols show the maxima of the premultiplied energy spectra $\max \left(k_{1}^{+} \tilde{E}_{i i}^{+}\right)$. The hollow triangle in (c) shows the secondary peak of $k_{1}^{+} \tilde{E}_{33}^{+}$. The dashed-dotted lines represent the isopleths of $k_{1}^{+} \tilde{E}_{i i}^{+}=0.5 \max \left(k_{1}^{+} \tilde{E}_{i i}^{+}\right)$and $k_{1}^{+} \tilde{E}_{i i}^{+}=0.85 \max \left(k_{1}^{+} \tilde{E}_{i i}^{+}\right)$. Here, the summation convection over subscript $i$ is not applied. The wave-number $\lambda_{1}$ is shown in a logarithmic coordinate.

The premultiplied energy spectra $k_{1}^{+} \tilde{E}_{i i}^{+}$is a useful tool for diagnosing the streamwise scale of the vortex structures, where $\tilde{E}_{i i}\left(k_{1}, x_{2}\right)=\operatorname{Re}\left\{\widehat{u_{i}^{*} * \widehat{u_{i}^{\prime}}}\right\}$ is the streamwise energy spectrum of $u_{i}^{\prime}$. Note that the summation convention over subscript " $i$ " is not applied. The hat denotes the Fourier coefficient of an arbitrary variable $\phi\left(x_{1}, x_{2}, x_{3}, t\right)$ as

$$
\hat{\phi}\left(k_{1}, x_{2}, x_{3}, t\right)=\frac{1}{L_{1}} \int_{0}^{L_{1}} \phi\left(x_{1}, x_{2}, x_{3}, t\right) e^{-i k_{1} x_{1}} d x_{1},
$$

where $i=\sqrt{-1}$ is the imaginary unit and $k_{1}=n_{1} k_{01}$ is the streamwise wave number with $n_{1} \in$ $\left[-N_{1} / 2, N_{1} / 2-1\right]$ being an integer and $k_{01}=2 \pi / L_{1}$ being the lowest positive wave number in the streamwise direction. The superscript ${ }^{*}$ and operator $\operatorname{Re}\{\}$ denote the conjugate and real part of a complex number, respectively. The overline represents averaging over time $t$ and spanwise direction $x_{3}$.

Figure 3 displays the contours of $k_{1}^{+} \tilde{E}_{11}^{+}, k_{1}^{+} \tilde{E}_{22}^{+}$, and $k_{1}^{+} \tilde{E}_{33}^{+}$at $\operatorname{Ro}_{\tau}=150$ on a $\lambda_{1}-x_{2}$ plane where the streamwise wavelength $\lambda_{1}$ is defined as $\lambda_{1}=2 \pi / k_{1}$. The wave-number $\lambda_{1}$ is shown in a logarithmic coordinate in all figures of this paper. As shown using the cross symbol in Figs. 3(a) and $3(\mathrm{c})$, the peaks of $k_{1}^{+} \tilde{E}_{11}^{+}$and $k_{1}^{+} \tilde{E}_{33}^{+}$both occur at $x_{2} / h=-0.9$, whereas in Fig. $3(\mathrm{~b})$, the maximum 

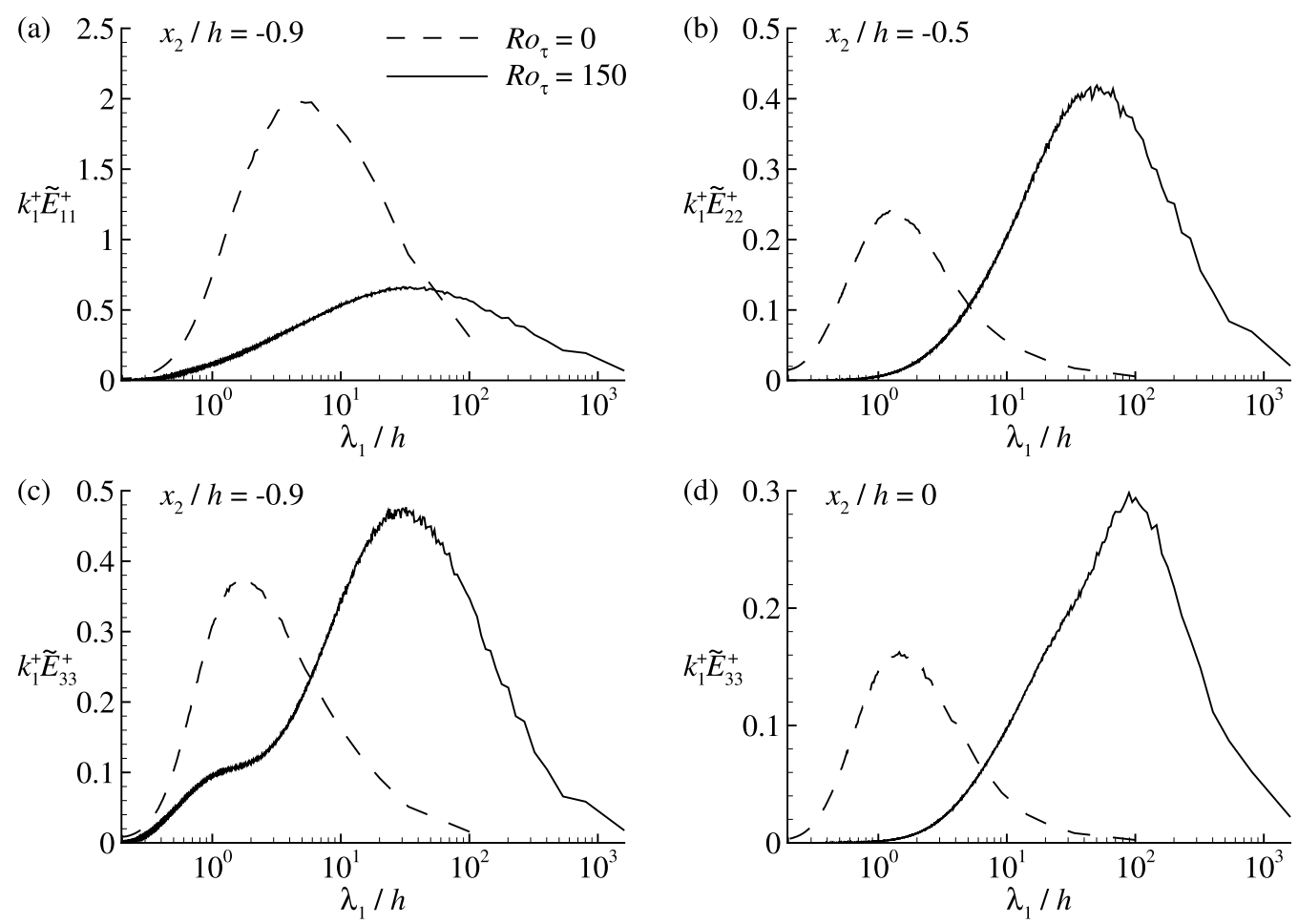

FIG. 4. Profiles of premultiplied energy spectra $k_{1}^{+} \tilde{E}_{i j}^{+}$in nonrotating $\left(\mathrm{Ro}_{\tau}=0\right)$ and streamwise-rotating $\left(\operatorname{Ro}_{\tau}=150\right)$ channel flows. (a) $k_{1}^{+} \tilde{E}_{11}^{+}$at $x_{2} / h=-0.9$, (b) $k_{1}^{+} \tilde{E}_{22}^{+}$at $x_{2} / h=-0.5$, (c) $k_{1}^{+} \tilde{E}_{33}^{+}$at $x_{2} / h=-0.9$, and (d) $k_{1}^{+} \tilde{E}_{33}^{+}$at $x_{2} / h=0$. The wave-number $\lambda_{1}$ is shown in a logarithmic coordinate.

of $k_{1}^{+} \tilde{E}_{22}^{+}$is located at $x_{2} / h=-0.5$. The contours of $k_{1}^{+} \tilde{E}_{33}^{+}$also show a secondary peak at the channel center $\left(x_{2} / h=0\right)$. These peaks of $k_{1}^{+} \tilde{E}_{i j}^{+}$all occur at large wavelengths ranging from $\lambda_{1} / h=$ 30 to 90 , which can be regarded as characteristic length scales of the TGL vortices. The pattern of $k_{1}^{+} \tilde{E}_{i j}^{+}$shown in Fig. 3 is typical for large-scale streamwise vortical motions. The wall-normal location $x_{2} / h=-0.5$ of the maximum of $k_{1}^{+} \tilde{E}_{22}^{+}$indicates the averaged location of the core of the TGL vortices, whereas the large-scale spanwise motions are enhanced at the bottom and top edges of the TGL vortices, located around $x_{2} / h=-0.9$ and $x_{2} / h=0$, respectively. The wall-normal location of the TGL vortices diagnosed from the energy spectra on the $\lambda_{1}-x_{2}$ plane is consistent with the observation of the instantaneous TGL vortices in the physical space shown in Fig. 1.

To further investigate the effect of the TGL vortices on the energy spectra, the profiles of the premultiplied energy spectra $k_{1}^{+} \tilde{E}_{i i}^{+}$in the nonrotating and streamwise-rotating channel flows are compared in Fig. 4. To focus on the characteristics of the TGL vortices inferred from Fig. 3, the results are shown at the wall-normal positions corresponding to the peaks of $k_{1}^{+} \tilde{E}_{i i}^{+}$at $\operatorname{Ro}_{\tau}=150 \mathrm{in}$ Fig. 3. Specifically, the profiles of $k_{1}^{+} \tilde{E}_{11}^{+}$and $k_{1}^{+} \tilde{E}_{22}^{+}$are shown at $x_{2} / h=-0.9$ and $x_{2} / h=-0.5$, respectively, whereas those of $k_{1}^{+} \tilde{E}_{33}^{+}$are shown at $x_{2} / h=-0.9$ and 0 . It is seen from Fig. 4(a) that the peak of $k_{1}^{+} \tilde{E}_{11}^{+}$occurs at a large wavelength [Fig. 3(a)] in the streamwise-rotating channel flow. It is shown in Sec. IV that this is mainly because of the suppression of $k_{1}^{+} \tilde{E}_{11}^{+}$at small wavelengths in response to the imposed streamwise system rotation. Figures 4(b)-4(d) show that the magnitudes of $k_{1}^{+} \tilde{E}_{22}^{+}$and $k_{1}^{+} \tilde{E}_{33}^{+}$at large wavelengths is higher at $\operatorname{Ro}_{\tau}=150$ than at $\operatorname{Ro}_{\tau}=0$. These observations of $k_{1}^{+} \tilde{E}_{22}^{+}$and $k_{1}^{+} \tilde{E}_{33}^{+}$directly reflect the characteristics of the TGL vortices, i.e., the strong motions in a cross-stream plane consisting of wall-normal and spanwise velocity fluctuations at large streamwise length scales. 
From the above examinations of the instantaneous field and turbulence statistics of a streamwiserotating channel flow, we can summarize the following four distinct features of the energy spectra characterizing the motions of TGL vortices. Given the symmetry of the channel in the wall-normal direction and the presence of one layer of TGL vortices on each side of the channel, this summary is based on the lower half of the channel (for $-1 \leqslant x_{2} / h \leqslant 0$ ). There exist:

(i) the suppression of $k_{1}^{+} \tilde{E}_{11}^{+}$at relatively small streamwise wavelengths (for $\lambda_{1} / h<50$ ) in the near-wall region $\left(x_{2} / h=-0.9\right)$;

(ii) the enhancement of $k_{1}^{+} \tilde{E}_{22}^{+}$at large streamwise wavelengths (peaking at $\lambda_{1} / h \approx 50$ ) around the core region of the TGL vortices $\left(x_{2} / h=-0.5\right)$;

(iii) the enhancement of $k_{1}^{+} \tilde{E}_{33}^{+}$at large streamwise wavelengths (peaking at $\lambda_{1} / h \approx 30$ ) around the bottom edge of the TGL vortices $\left(x_{2} / h=-0.9\right)$; and

(iv) the enhancement of $k_{1}^{+} \tilde{E}_{33}^{+}$at large streamwise wavelengths (peaking at $\lambda_{1} / h \approx 90$ ) around the top edge of the TGL vortices $\left(x_{2} / h=0\right)$.

These four features of the energy spectra are further investigated in Sec. IV through the analyses of their transport equations.

\section{ENERGY TRANSPORT PROCESSES FOR SUSTAINING TGL VORTICES}

To develop deeper insights into the sustaining mechanism of the TGL vortices, it is useful to study the energy transport processes at different characteristic length scales. For this purpose, we study the transport equation of velocity-spectrum tensor, expressed as (see the Appendix for the derivation)

$$
\frac{\partial \tilde{E}_{i j}}{\partial t}=0=\tilde{P}_{i j}+\tilde{\Gamma}_{i j}^{r}+\tilde{G}_{i j}^{r}+\tilde{\Gamma}_{i j}^{c}+\tilde{G}_{i j}^{c}+\tilde{\varepsilon}_{i j}+\tilde{D}_{i j}+\tilde{T}_{i j}^{p}+\tilde{T}_{i j}^{s}
$$

The budget terms on the right-hand side of (4) include the production term $\tilde{P}_{i j}$, rotation-induced redistribution term $\tilde{\Gamma}_{i j}^{r}$, rotation-induced pressure-diffusion term $\tilde{G}_{i j}^{r}$, convection-induced redistribution term $\tilde{\Gamma}_{i j}^{c}$, convection-induced pressure-diffusion term $\tilde{G}_{i j}^{c}$, dissipation term $\tilde{\varepsilon}_{i j}$, viscousdiffusion term $\tilde{D}_{i j}$, wall-normal turbulence-diffusion term $\tilde{T}_{i j}^{p}$, and the interscale transport term $\tilde{T}_{i j}^{s}$, which are, respectively, defined as

$$
\begin{aligned}
& \tilde{P}_{i j}=\operatorname{Re}\left\{-\widehat{u_{j}^{\prime} \widehat{u}_{2}^{*}} \frac{\partial\left\langle u_{i}\right\rangle}{\partial x_{2}}-\overline{u_{i}^{\prime} * \widehat{u}_{2}^{\prime}} \frac{\partial\left\langle u_{j}\right\rangle}{\partial x_{2}}\right\},
\end{aligned}
$$

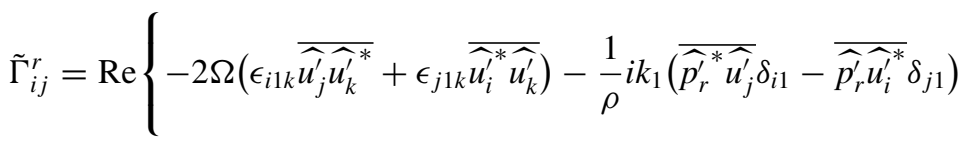

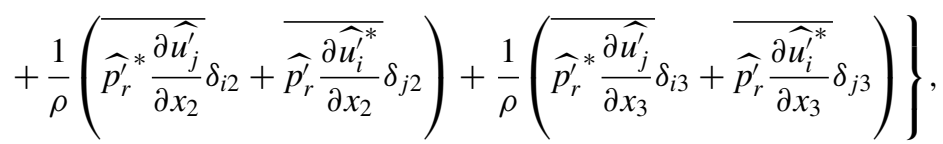

$$
\begin{aligned}
& \tilde{G}_{i j}^{r}=\operatorname{Re}\left\{-\frac{1}{\rho}\left(\frac{\partial \widehat{{\widehat{p_{r}^{\prime}}}^{*}{\widehat{u_{j}^{\prime}}}_{j}}}{\partial x_{2}} \delta_{i 2}+\frac{\partial \widehat{\widehat{p}_{r}^{\prime}{\widehat{u_{i}^{\prime}}}^{*}}}{\partial x_{2}} \delta_{j 2}\right)\right\},
\end{aligned}
$$

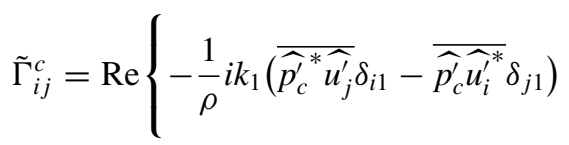

$$
\begin{aligned}
& \left.+\frac{1}{\rho}\left(\widehat{{\widehat{p_{c}^{\prime}}}_{c}^{*} \frac{\partial \widehat{u}_{j}^{\prime}}{\partial x_{2}}} \delta_{i 2}+\widehat{\widehat{p_{c}^{\prime}} \frac{\partial \widehat{u}_{i}^{\prime *}}{\partial x_{2}}} \delta_{j 2}\right)+\frac{1}{\rho}\left(\widehat{{\widehat{p_{c}^{\prime}}}_{c}^{*} \frac{\partial \widehat{u}_{j}^{\prime}}{\partial x_{3}}} \delta_{i 3}+\widehat{\hat{p}_{c}^{\prime} \frac{\partial \widehat{u}_{i}^{*}}{\partial x_{3}}} \delta_{j 3}\right)\right\},
\end{aligned}
$$




$$
\begin{aligned}
& \tilde{G}_{i j}^{c}=\operatorname{Re}\left\{-\frac{1}{\rho}\left(\frac{\partial \widehat{\widehat{p}_{c}^{\prime *} \widehat{u}_{j}^{\prime}}}{\partial x_{2}} \delta_{i 2}+\frac{\partial \widehat{\widehat{p}_{c}^{\prime}{\widehat{u_{i}^{\prime}}}^{*}}}{\partial x_{2}} \delta_{j 2}\right)\right\}, \\
& \tilde{\varepsilon}_{i j}=\operatorname{Re}\left\{-2 v\left(k_{1}^{2} \widehat{\widehat{u}_{i}^{\prime} \widehat{u_{j}^{\prime}}}+\overline{\frac{\partial \widehat{u}_{i}^{\prime}}{\partial x_{2}} \frac{\partial \widehat{u}_{j}^{\prime}}{\partial x_{2}}}+\overline{\frac{\partial \widehat{u}_{i}^{\prime}}{\partial x_{3}} \frac{\partial \widehat{u}_{j}^{\prime}}{\partial x_{3}}}\right)\right\}, \\
& \tilde{D}_{i j}=\operatorname{Re}\left\{v \frac{\partial^{2} \widehat{\widehat{u_{i}^{*}} \widehat{u_{j}^{\prime}}}}{\partial x_{2}^{2}}\right\} \text {, } \\
& \tilde{T}_{i j}^{p}=\operatorname{Re}\left\{-\frac{1}{2}\left(\frac{\partial \widehat{{\hat{u_{i}^{\prime} u_{2}^{\prime}}}^{*}{\widehat{u_{j}^{\prime}}}_{j}}}{\partial x_{2}}+\frac{\partial \widehat{u_{j}^{\prime} u_{2}^{\prime}}{\widehat{u_{i}^{\prime}}}^{*}}{\partial x_{2}}\right)\right\}
\end{aligned}
$$

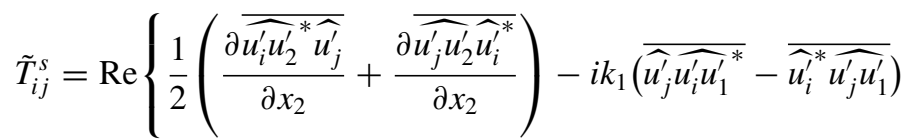

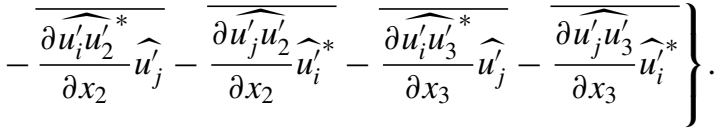

In Eqs. (6)-(9), $p_{c}^{\prime}$ and $p_{r}^{\prime}$ denote convection-induced pressure and rotation-induced pressure fluctuations, respectively. The decomposition of the pressure fluctuations follows the decomposition of pressure $p=p_{c}+p_{r}$ proposed by Yang et al. [16], which separates the nonrotating and rotating effects on the pressure field. The decomposition procedure starts by taking the divergence of Eq. (2) and utilizing the continuity equation to obtain

$$
\frac{1}{\rho} \frac{\partial^{2} p}{\partial x_{i} \partial x_{i}}=-\frac{\partial u_{i}}{\partial x_{j}} \frac{\partial u_{j}}{\partial x_{i}}+2 \Omega \omega_{1},
$$

where $\omega_{1}=\partial u_{3} / \partial x_{2}-\partial u_{2} / \partial x_{3}$ is the streamwise vorticity. Equation (14) can be further linearly decomposed into two equations that govern the convection-induced and rotation-induced pressure fields, respectively. The convection-induced pressure $p_{c}$ is governed by the following Poisson equation and boundary condition:

$$
\frac{1}{\rho} \frac{\partial^{2} p_{c}}{\partial x_{i} \partial x_{i}}=-\frac{\partial u_{i}}{\partial x_{j}} \frac{\partial u_{j}}{\partial x_{i}} \quad \text { with } \quad \frac{\partial p_{c}}{\partial x_{2}}=\rho v \frac{\partial^{2} u_{2}}{\partial x_{2}^{2}} \quad \text { at } \quad x_{2}= \pm h,
$$

whereas the Poisson equation and boundary condition for the rotation-induced pressure $p_{r}$ are expressed as

$$
\frac{1}{\rho} \frac{\partial^{2} p_{r}}{\partial x_{i} \partial x_{i}}=2 \Omega \omega_{1} \quad \text { with } \quad \frac{\partial p_{r}}{\partial x_{2}}=0 \quad \text { at } \quad x_{2}= \pm h
$$

The above linear decomposition method for the pressure is unique mathematically as there is a natural constraint that requires the rotation-induced pressure $p_{r}$ to vanish in the context of a nonrotating flow, i.e., $\partial p_{r} / \partial x_{i}=0$ if $\Omega=0$. By applying the decomposition of pressure fluctuations, two new terms, namely, the rotation-induced redistribution term $\tilde{\Gamma}_{i j}^{r}$ and rotation-induced pressure-diffusion term $\tilde{G}_{i j}^{r}$ are defined to capture the effect of system rotation on the energy transport process. The physical processes represented by $\tilde{\Gamma}_{i j}^{r}$ and $\tilde{G}_{i j}^{r}$ are clear. The function of $\tilde{\Gamma}_{i j}^{r}$ is to redistribute energy among the three normal components of the velocity-spectrum tensor because $\tilde{\Gamma}_{i i}^{r}=0$ holds strictly. 
The function of $\tilde{G}_{i j}^{r}$ is to transport energy in the wall-normal direction because the integration of $\tilde{G}_{i j}^{r}$ over $x_{2}$ is zero, i.e., $\int_{-h}^{h} G_{i j}^{r} d x_{2}=0$.

The rotation-induced redistribution term $\tilde{\Gamma}_{i j}^{r}$ in Eq. (7) consists of two parts, namely, the contributions from the Coriolis force $\tilde{\Gamma}_{i j}^{r c}$ and the rotation-induced pressure $\tilde{\Gamma}_{i j}^{r p}$, defined, respectively, as

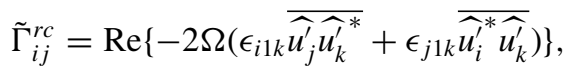

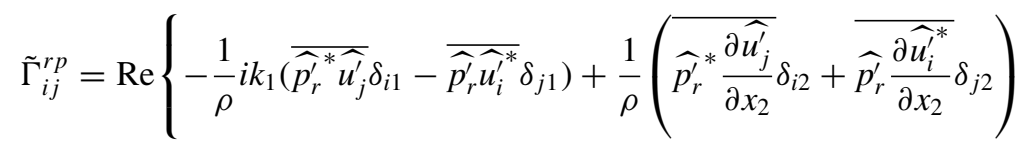

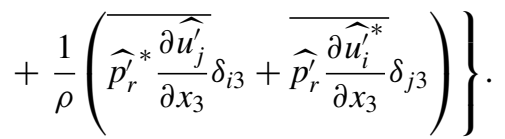

Figure 5 compares the profiles of $k_{1}^{+} \tilde{\Gamma}_{i j}^{r+}, k_{1}^{+} \tilde{\Gamma}_{i j}^{r c+}$, and $k_{1}^{+} \tilde{\Gamma}_{i j}^{r p+}$ to demonstrate the purpose of considering $\tilde{\Gamma}_{i j}^{r}$ as a whole in the present analyses of the energy spectra budgets. In consistency with Fig. 4 , the results of the 11-component at $x_{2} / h=-0.9,22$-component at $x_{2} / h=-0.5$, and 33-component at $x_{2} / h=-0.9$ and 0 are shown in Fig. 5. Because the magnitudes of $k_{1}^{+} \tilde{\Gamma}_{22}^{r+}$ and $k_{1}^{+} \tilde{\Gamma}_{33}^{r+}$ are significantly smaller than those of their corresponding two parts, the values of $k_{1}^{+} \tilde{\Gamma}_{22}^{r+}$ and $k_{1}^{+} \tilde{\Gamma}_{33}^{r+}$ in Figs. 5(b) and 5(c) are amplified by a factor of 10 , whereas the value of $k_{1}^{+} \tilde{\Gamma}_{33}^{r+}$ in Fig. 5(d) is amplified by a factor of 50 to ensure a clearer visualization. The value of $\tilde{\Gamma}_{11}^{r c+}$ is zero by definition in Eq. (17). As a result, the profile of $\tilde{\Gamma}_{11}^{r+}$ overlaps that of $\tilde{\Gamma}_{11}^{r p+}$ in Fig. 5(a). In the budget balance of $\tilde{E}_{22}^{+}$and $\tilde{E}_{33}^{+}$as shown in Figs. 5(b)-5(d), the behaviors of $\tilde{\Gamma}_{i j}^{r+}$ and $\tilde{\Gamma}_{i j}^{r p+}$ are opposite each other, indicating that their effects on the transport of energy spectra are mostly canceled out. As a result, the net rotation-induced redistribution effect as represented by $\tilde{\Gamma}_{i j}^{r+}$ is actually much smaller than that of either $\tilde{\Gamma}_{i j}^{r c+}$ or $\tilde{\Gamma}_{i j}^{r p+}$. As such, the effect of the system rotation on the transport of energy spectra can be misidentified or exaggerated if only the contribution of the Coriolis force is considered. For example, as shown in Figs. 5(c) and 5(d), if the attention is solely paid to the negatively valued $\tilde{\Gamma}_{33}^{r c+}$, it leads to an erroneous conclusion that the system rotation tends to suppress the spanwise velocity fluctuations at all streamwise wavelengths. On the contrary, the value of the rotation-induced redistribution term $\tilde{\Gamma}_{33}^{r+}$ is positive at both small and large wavelengths, indicating that the net effect of the streamwise system rotation is actually to intensify the spanwise velocity fluctuations. Furthermore, it is seen from Figs. 5(b)-5(d) that the profiles of $\tilde{\Gamma}_{22}^{r c+}, \tilde{\Gamma}_{22}^{r p+}, \tilde{\Gamma}_{33}^{r c+}$, and $\tilde{\Gamma}_{33}^{r p+}$ show only one peak, but the profiles of both $\tilde{\Gamma}_{22}^{r+}$ and $\tilde{\Gamma}_{33}^{r+}$ form bimodal shapes. This indicates that if $\tilde{\Gamma}_{i j}^{r c+}$ and $\tilde{\Gamma}_{i j}^{r p+}$ are treated as two independent effects in the budget analyses, the energy redistribution at certain wavelengths would be overlooked. For example, in Fig. 5(c), both $\tilde{\Gamma}_{33}^{r c+}$ and $\tilde{\Gamma}_{33}^{r p+}$ peak at a relatively small wavelength $\lambda_{1} / h=1.6$, but $\tilde{\Gamma}_{33}^{r+}$ shows an additional peak at a much larger wavelength $\lambda_{1} / h=30$. In other words, considering $\tilde{\Gamma}_{33}^{r c+}$ and $\tilde{\Gamma}_{33}^{r p+}$ separately would miss the energy gain by $\tilde{E}_{33}$ at large scales through the rotation-induced energy redistribution.

Figure 6 compares the premultiplied budget terms in the transport equation of $\tilde{E}_{11}^{+}$for the nonrotating $\left(\mathrm{Ro}_{\tau}=0\right)$ and streamwise-rotating $\left(\operatorname{Ro}_{\tau}=150\right)$ channel flows at $x_{2} / h=-0.9$. Given the fact that the streamwise computational domain size is much larger at $\operatorname{Ro}_{\tau}=150$ than at $\operatorname{Ro}_{\tau}=0$ (Table II), the range of the nondimensionalized streamwise wavelength $\lambda_{1} / h$ is kept the same in Figs. 6(a) and 6(b) to make a direct comparison of the characteristic length scales of the budget terms at these two rotation numbers. Specifically, $\lambda_{1}$ ranges from $\pi h / 16$ (twice the grid resolution in the $x_{1}$ direction at both rotation numbers) to $512 \pi h$ (streamwise computational domain size for the case of $\mathrm{Ro}_{\tau}=150$ ). The streamwise wavelength corresponding to the peak of $k_{1}^{+} \tilde{E}_{11}^{+}$is shown 

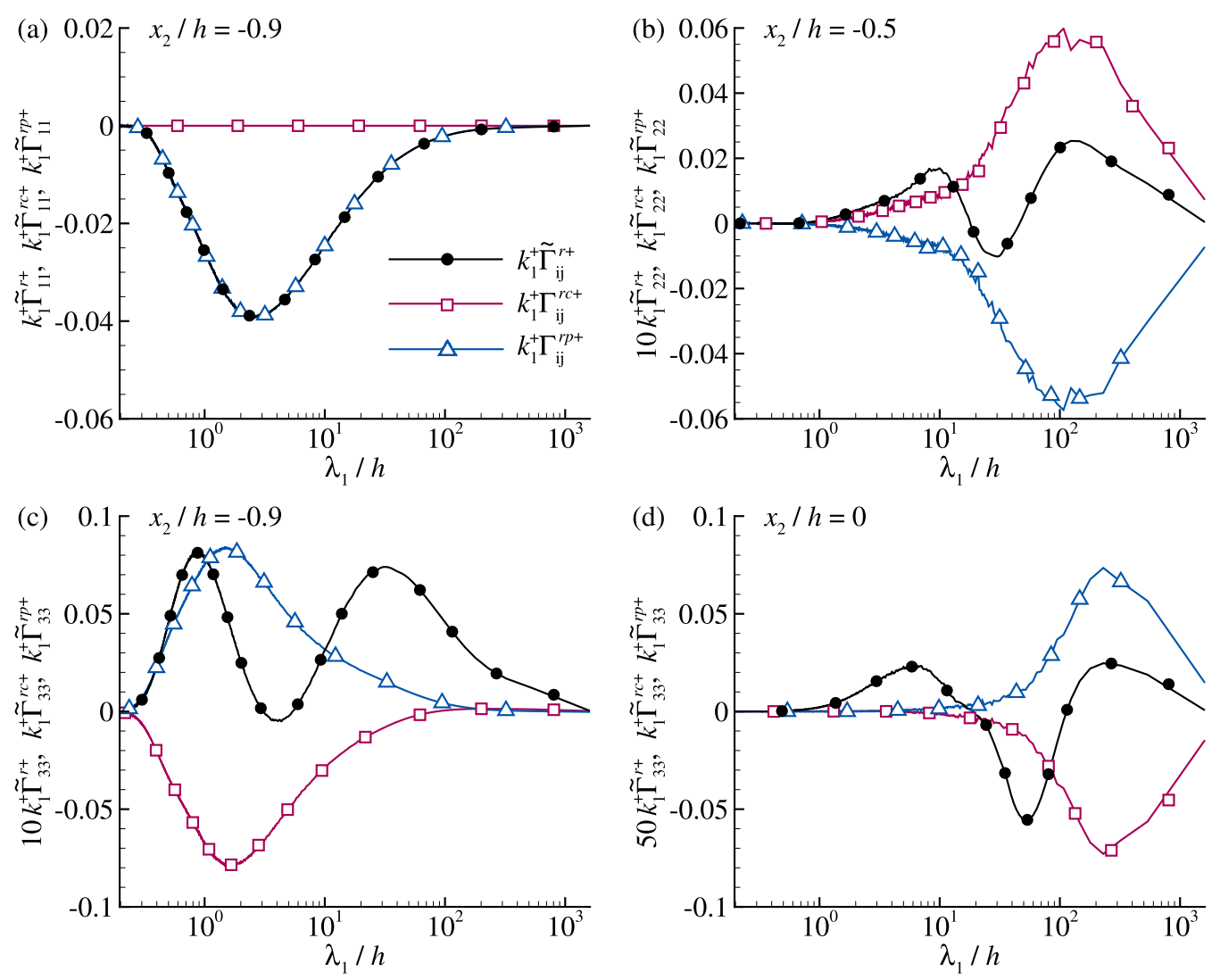

FIG. 5. Premultiplied rotation-induced redistribution term $k_{1}^{+} \tilde{\Gamma}_{i j}^{r+}$ and its two parts $k_{1}^{+} \tilde{\Gamma}_{i j}^{r c+}$ and $k_{1}^{+} \tilde{\Gamma}_{i j}^{r p+}$ at $\operatorname{Ro}_{\tau}=150$. (a) The 11-component at $x_{2} / h=-0.9$, (b) 22-component at $x_{2} / h=-0.5$, (c) 33-component at $x_{2} / h=-0.9$, and (d) 33-component at $x_{2} / h=0$. Because the magnitudes of $k_{1}^{+} \tilde{\Gamma}_{22}^{r+}$ and $k_{1}^{+} \tilde{\Gamma}_{33}^{r+}$ are significantly smaller than those of their corresponding two parts, the values of $k_{1}^{+} \tilde{\Gamma}_{22}^{r+}$ and $k_{1}^{+} \tilde{\Gamma}_{33}^{r+}$ in panels (b) and (c) are amplified by a factor of 10 , whereas the value of $k_{1}^{+} \tilde{\Gamma}_{33}^{r+}$ in panel (d) is amplified by a factor of 50 to ensure a clearer visualization. The wave-number $\lambda_{1}$ is shown in a logarithmic coordinate.

in Fig. 6(b) using the vertical dashed-dotted line as a reference that demarcates the characteristic length scale of the TGL vortices.

Figure 6 shows two mechanisms in the streamwise-rotating channel flow that dominate the suppression of $k_{1}^{+} \tilde{E}_{11}^{+}$at relatively small length scales (Fig. 4). The first mechanism is associated with the reduction of energy production. From the comparison between Figs. 6(a) and 6(b), it is seen that the wavelengths corresponding to the peaks of $k_{1}^{+} \tilde{P}_{11}^{+}$in the nonrotating and streamwise-rotating channel flows are close. This indicates that the energy production in the streamwise-rotating channel flow is dominated by the near-wall vortex structures as in the nonrotating channel flow. However, the peak value of $k_{1}^{+} \tilde{P}_{11}^{+}$is smaller in the streamwise-rotating channel flow than in the nonrotating channel flow. This is partially responsible for the energy suppression at small scales in the streamwise-rotating channel flow. In closely relevant to the present paper, the energy suppression at small scales is also observed in the spanwise-rotating channel flow. As noted by Kawata and Alfredsson [9], in the spanwise-rotating channel flow, the reduction of energy production at small scales is the dominant reason for the suppression of near-wall vortex structures. The second mechanism underlying the suppression of $k_{1}^{+} \tilde{E}_{11}^{+}$at small length scales is the rotation-induced redistribution term. As shown in Fig. 6(a), in the nonrotating channel flow, the dissipation term $\tilde{\varepsilon}_{11}^{+}$ 

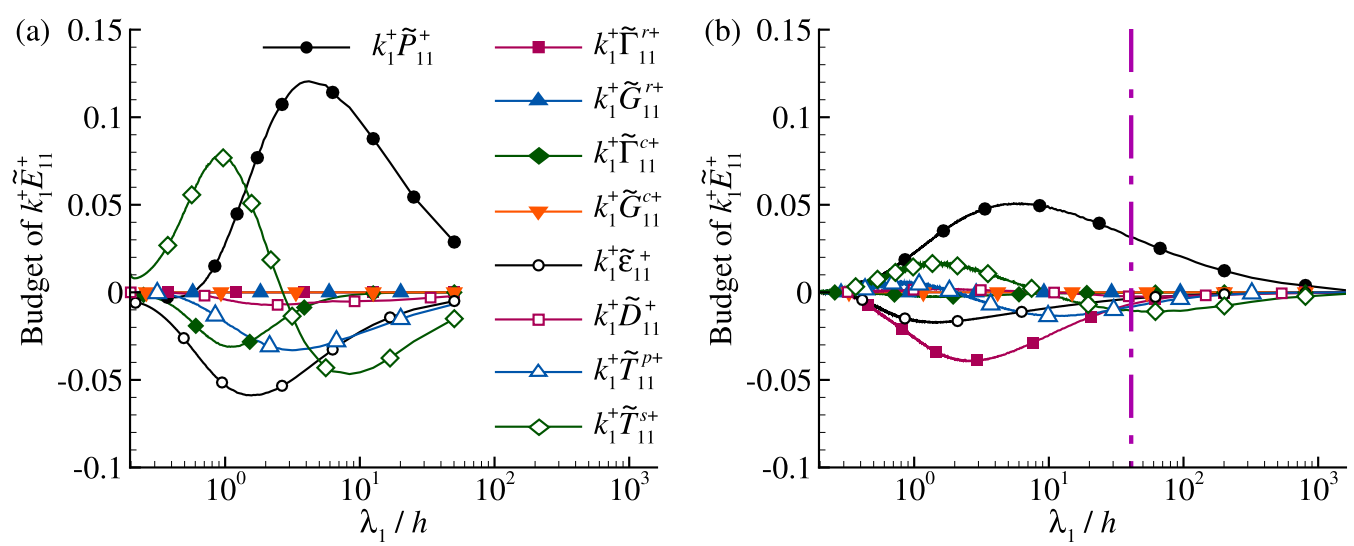

FIG. 6. Premultiplied budget terms in the transport equation of spectrum $\tilde{E}_{11}^{+}$at $x_{2} / h=-0.9$ for (a) nonrotating $\left(\mathrm{Ro}_{\tau}=0\right)$ and (b) streamwise-rotating $\left(\mathrm{Ro}_{\tau}=150\right)$ turbulent channel flows. The vertical dashed-dotted line demarcates the characteristic streamwise wavelength corresponding to the maximum value of $k_{1}^{+} \tilde{E}_{11}^{+}$ [marked by the cross symbol in Fig. 3(a)]. The wave-number $\lambda_{1}$ is shown in a logarithmic coordinate.

is the dominant sink term, but Fig. 6(b) shows that, in the streamwise-rotating channel flow, most energy is consumed by the rotation-induced redistribution term $\tilde{\Gamma}_{11}^{r+}$, indicating that the effect of streamwise system rotation tends to transfer more energy from the streamwise velocity fluctuations to the wall-normal and spanwise velocity fluctuations. The rotation-induced redistribution term is also responsible for the significant difference in the wavelengths corresponding to the peaks of $k_{1}^{+} \tilde{P}_{11}^{+}$and $k_{1}^{+} \tilde{E}_{11}^{+}$. It is seen from the comparison between Figs. 4(a) and 6(b) that the peak of $k_{1}^{+} \tilde{E}_{11}^{+}$occurs at $\lambda_{1} / h=40$, which is much larger than the wavelength corresponding to the peak of $k_{1}^{+} \tilde{P}_{11}^{+}$. Meanwhile, Fig. 6(b) shows no additional energy production mechanism at $\lambda_{1} / h=40$ that induces the peak of $k_{1}^{+} \tilde{E}_{11}^{+}$there. Therefore, it is evident that the additional energy loss at small wavelengths due to the rotation-induced redistribution leads to the peak shift of $k_{1}^{+} \tilde{E}_{11}^{+}$to a much larger wavelength.

To further investigate the contributions of the production term and rotation-induced redistribution term to the sustaining of the TGL vortices in the streamwise-rotating channel flow, contours of $k_{1}^{+} \tilde{P}_{11}^{+}$ and $k_{1}^{+} \tilde{\Gamma}_{11}^{r+}$ on a $\lambda_{1}-x_{2}$ plane are displayed in Fig. 7. The isopleth of $k_{1}^{+} \tilde{E}_{11}^{+}=0.85 \max \left(k_{1}^{+} \tilde{E}_{11}^{+}\right)$is superimposed for the purpose of comparison, which shows the ranges of streamwise wavelength and wall-normal location where the TGL vortices are energetic. It is seen that on the $\lambda_{1}-x_{2}$ plane, the region with large magnitude of $k_{1}^{+} \tilde{E}_{11}^{+}$(inside the dashed-dotted line) collocates with the regions with large energy gain through the production term $\tilde{P}_{11}^{+}$and relatively small energy loss through the rotation-induced redistribution term $\tilde{\Gamma}_{11}^{r+}$ [in comparison with that at small wavelengths outside the dashed-dotted line in Fig. 7(b)]. This observation, together with the result of $k_{1}^{+} \tilde{E}_{11}^{+}$ shown in Fig. 4(a), indicates that it is the differences in the energy gain through $\tilde{P}_{11}^{+}$and in the energy loss through $\tilde{\Gamma}_{11}^{r+}$ that determine the energy level of $\tilde{E}_{11}^{+}$. To be specific, at relatively small wavelengths $\left(\lambda_{1} / h<50\right)$ in the near-wall region, the summation of $k_{1}^{+} \tilde{P}_{11}^{+}$and $k_{1}^{+} \tilde{\Gamma}_{11}^{r+}$ is smaller in the streamwise-rotating channel flow than in the nonrotating channel flow, resulting in the suppression of $k_{1}^{+} \tilde{E}_{11}^{+}$[i.e., feature (i) of the energy spectra summarized in Sec. III].

It is also found that at $x_{2} / h=-0.5$ (where the cores of the TGL vortices are concentrated), the maximum value of $k_{1}^{+} \tilde{P}_{11}^{+}$occurs at a large wavelength of $\lambda_{1} / h \approx 100$, indicating a self-enhancement behavior of the TGL vortices. Once the TGL vortices appear in the streamwise-rotating channel flow, they induce energy production at large length scales to sustain the motions of themselves. Based on the budget analyses of $\tilde{E}_{22}^{+}$, it is shown below that the energy production at large length scales in $\tilde{E}_{11}^{+}$is further transferred to $\tilde{E}_{22}^{+}$through the rotation-induced energy redistribution process, leading to the enhancement of the wall-normal velocity fluctuations. 

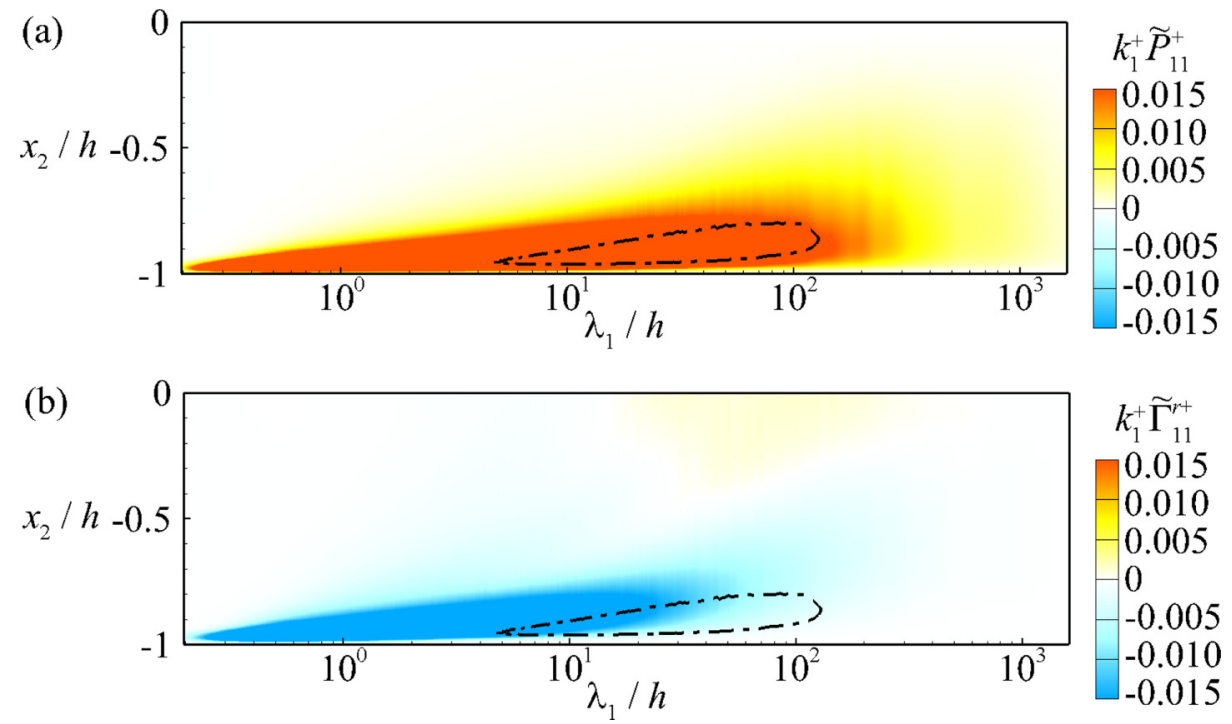

FIG. 7. Contours of key premultiplied budget terms in the transport equation of $\tilde{E}_{11}^{+}$on a $\lambda_{1}-x_{2}$ plane at $\mathrm{Ro}_{\tau}=150$. (a) Production term $k_{1}^{+} \tilde{P}_{11}^{+}$and (b) rotation-induced redistribution term $k_{1}^{+} \tilde{\Gamma}_{11}^{r+}$. The dashed-dotted line represents the isopleth of $k_{1}^{+} \tilde{E}_{11}^{+}=0.85 \max \left(k_{1}^{+} \tilde{E}_{11}^{+}\right)$. The wave-number $\lambda_{1}$ is shown in a logarithmic coordinate.

Figure 8 compares the premultiplied budget terms of $\tilde{E}_{22}^{+}$between the nonrotating and the streamwise-rotating channel flows at $x_{2} / h=-0.5$, which corresponds to the wall-normal location of $\max \left(k_{1}^{+} \tilde{E}_{22}^{+}\right)$on the $\lambda_{1}-x_{2}$ plane in the streamwise-rotating channel flow [Fig. 3(b)]. As shown in Fig. 8(a), in the nonrotating channel flow, the dominant source of $\tilde{E}_{22}^{+}$is the convection-induced redistribution term $\tilde{\Gamma}_{22}^{c+}$ for $\lambda_{1} / h>0.5$. The TKE is transported to smaller wavelengths by the interscale transport term $\tilde{T}_{22}^{s+}$ and then consumed by the dissipation term $\tilde{\varepsilon}_{22}^{+}$. In comparison with the nonrotating channel flow, the budget balance of $\tilde{E}_{22}^{+}$in the streamwise-rotating channel shown in Fig. 8(b) shows a more complex pattern. The rotation-induced redistribution term $\tilde{\Gamma}_{22}^{r+}$,
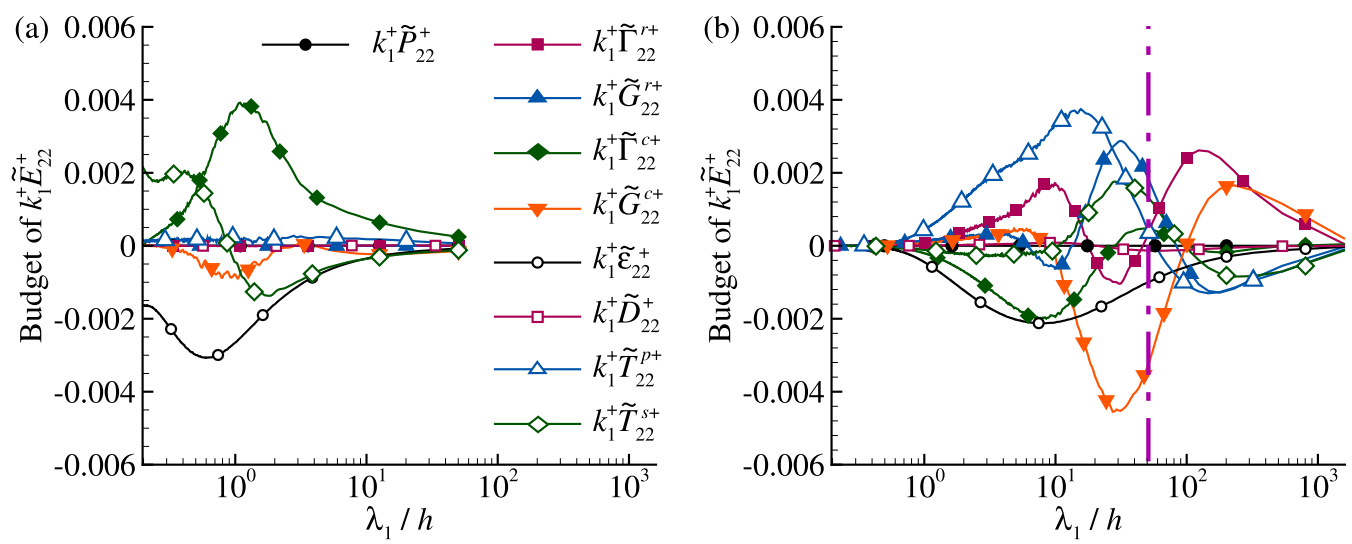

FIG. 8. Premultiplied budget terms in the transport equation of spectrum $\tilde{E}_{22}^{+}$at $x_{2} / h=-0.5$ for (a) nonrotating $\left(\mathrm{Ro}_{\tau}=0\right)$ and $(\mathrm{b})$ streamwise-rotating $\left(\mathrm{Ro}_{\tau}=150\right)$ turbulent channel flows. The vertical dashed-dotted line demarcates the characteristic streamwise wavelength corresponding to the maximum value of $k_{1}^{+} \tilde{E}_{22}^{+}$ [marked by the cross symbol in Fig. 3(b)]. The wave-number $\lambda_{1}$ is shown in a logarithmic coordinate. 
interscale transport term $\tilde{T}_{22}^{s+}$, wall-normal turbulent-diffusion term $\tilde{T}_{22}^{p+}$, rotation-induced pressurediffusion term $\tilde{G}_{22}^{r+}$, and convection-induced pressure-diffusion term $\tilde{G}_{22}^{c+}$ all act as sources at certain wavelengths. To further examine the contributions of these terms in sustaining the TGL vortices, the contours of their premultiplied values for the streamwise-rotating channel flow are displayed in Fig. 9.

From Fig. 9(b), it is seen that, on the $\lambda_{1}-x_{2}$ plane, the region featuring large magnitude of $k_{1}^{+} \tilde{E}_{22}^{+}$ (area enclosed by the dashed-dotted line) coincides with the region of positively valued interscale transport term $\tilde{T}_{22}^{s}$ (which represents an energy transport among different streamwise wavelengths at the same wall-normal location). From Fig. 8(b), it is seen that the energy gained by the wall-normal motions of the TGL vortices at $x_{2} / h=-0.5$ through $\tilde{T}_{22}^{s+}$ comes from both larger and smaller wavelengths for $\lambda_{1} / h>100$ and $\lambda_{1} / h<10$, respectively. This is different from the nonrotating channel flow where only energy transport from large to small wavelengths is observed from the budget balance of $k_{1}^{+} \tilde{E}_{22}^{+}$[Fig. 8(a)]. In other words, the TGL vortices are able to absorb energy from both larger and smaller eddies to sustain their own motions. Such an interscale energy transport process, especially the inverse one from small to large scales, is usually crucial for sustaining the large-scale vortex structures. For example, a similar inverse interscale energy transport process takes place in the turbulent Couette flow [33,34] to sustain large-scale vortex structures therein [25].

To further track the origin of the energy gain through the interscale energy transport process, we note that at the wavelengths smaller than the characteristic length scales of the TGL vortices (i.e., to the left of the region surrounded by the dashed-dotted line in Fig. 9), the dominant sources are the positively valued rotation-induced redistribution term $\tilde{\Gamma}_{22}^{r+}$ [Fig. 9(a)] and the wall-normal turbulent-diffusion term $\tilde{T}_{22}^{p+}$ [Fig. 9(c)]. The energy gain through $\tilde{\Gamma}_{22}^{r+}$ comes from the energy redistribution from the streamwise velocity fluctuations to the vertical ones at the same streamwise wavelength $\lambda_{1} / h$ and wall-normal location $x_{2} / h$. The function of $\tilde{T}_{22}^{p+}$ is to diffuse energy from the near-wall region at the same streamwise wavelengths, which also originates from the streamwise velocity fluctuations through a rotation-induced energy redistribution process [see the negatively valued $k_{1}^{+} \tilde{\Gamma}_{11}^{r+}$ in Fig. 7(b) and positively valued $k_{1}^{+} \tilde{\Gamma}_{22}^{r+}$ in Fig. 9(a)]. This indicates that the additional energy loss of $\tilde{E}_{11}^{+}$through the rotation-induced energy redistribution process in the near-wall region at small length scales [Fig. 4(a)] is eventually transferred to $\tilde{E}_{22}^{+}$at large length scales around $x_{2} / h=-0.5$ to sustain the motion of the TGL vortices. In other words, the suppression of the streamwise velocity fluctuations in the near-wall region is correlated to the energy supplying process of the TGL vortices. Furthermore, it is seen from Fig. 8 that the magnitude of the wall-normal redistribution term $\tilde{T}_{22}^{p+}$ is much larger in the streamwise-rotating channel flow than in the nonrotating channel flow, indicating that the TGL vortices are self-enhanced by raising the energy from the near-wall region to the channel center through a wall-normal turbulent diffusion process.

The energy production at large scales for $\lambda_{1} / h>50$ around $x_{2} / h=-0.5$ [as represented by the positively valued $k_{1}^{+} \tilde{P}_{11}^{+}$in Fig. 7(a)] also makes a contribution to the sustaining of the TGL vortices. As shown in Figs. 7(b) and 9(a), the values of the rotation-induced redistribution terms $k_{1}^{+} \tilde{\Gamma}_{11}^{r+}$ and $k_{1}^{+} \tilde{\Gamma}_{22}^{r+}$ are negative and positive, respectively, at the corresponding streamwise wavelength $\lambda_{1} / h$ and wall-normal location $x_{2} / h$. This indicates an energy transfer from $\tilde{E}_{11}^{+}$to $\tilde{E}_{22}^{+}$. Although the characteristic streamwise length scale of the rotation-induced energy redistribution process as represented by the peak of $k_{1}^{+} \tilde{\Gamma}_{22}^{r+}\left(\lambda_{1} / h \approx 100\right)$ is greater than that of $k_{1}^{+} \tilde{E}_{22}^{+}\left(\lambda_{1} / h \approx 50\right)$ at $x_{2} / h=$ -0.5 [Fig. 8(b)], this part of redistributed energy is eventually gained by the TGL vortices through the interscale energy transport process.

The roles of rotation-induced and convection-induced pressure-diffusion terms are opposite each other on the $\lambda_{1}-x_{2}$ plane within the region of $k_{1}^{+} \tilde{E}_{22}^{+} \geqslant 0.85 \max \left(k_{1}^{+} \tilde{E}_{22}^{+}\right)$, leaving a relatively small net contribution to the wall-normal motions of the TGL vortices. As shown in Figs. 9(d) and 9(e), the values of $k_{1}^{+} \tilde{G}_{22}^{r+}$ and $k_{1}^{+} \tilde{G}_{22}^{c+}$ are most positive and negative, respectively, at the corresponding $x_{2}$ locations and wavelengths $\lambda_{1} / h$ (inside the dashed-dotted line in the figure). The energy gain from the rotation-induced pressure-diffusion term mainly comes from near-wall $x_{2}$ locations with negative 

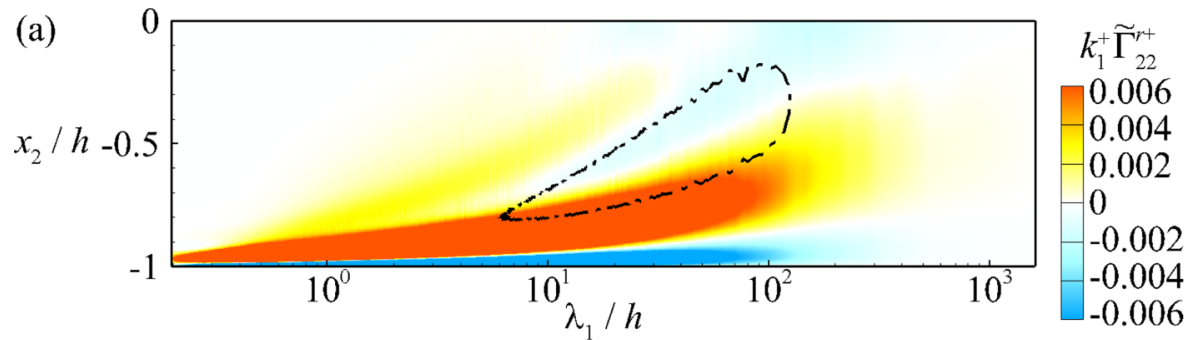

(b)
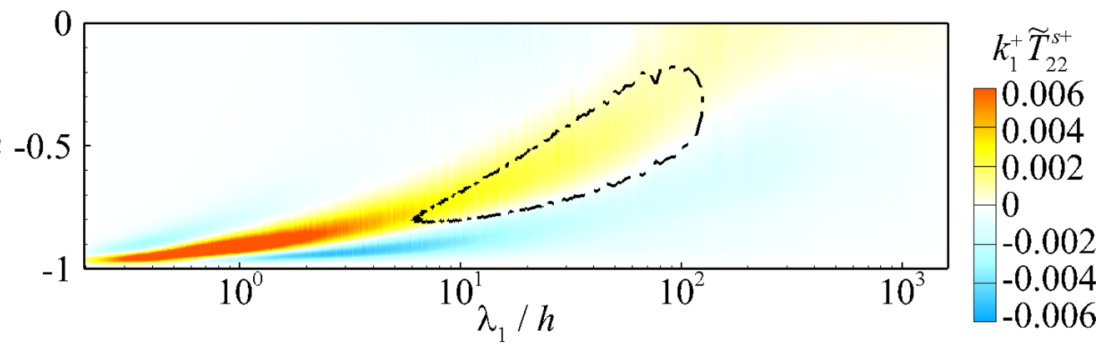

(c)
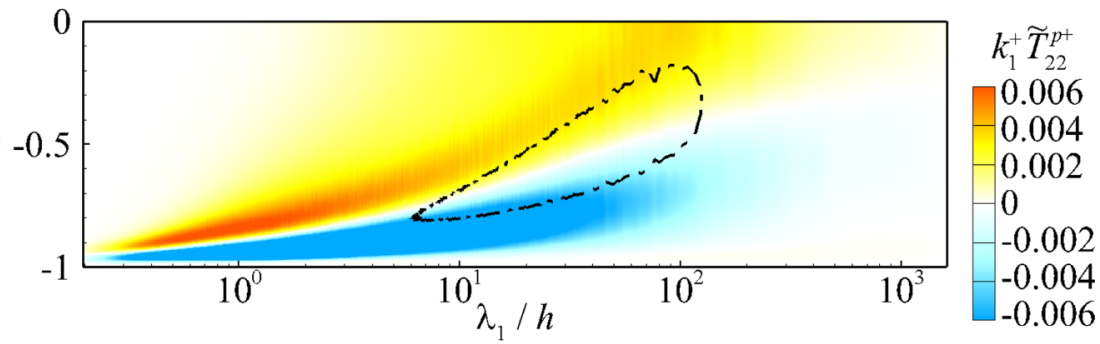

(d)
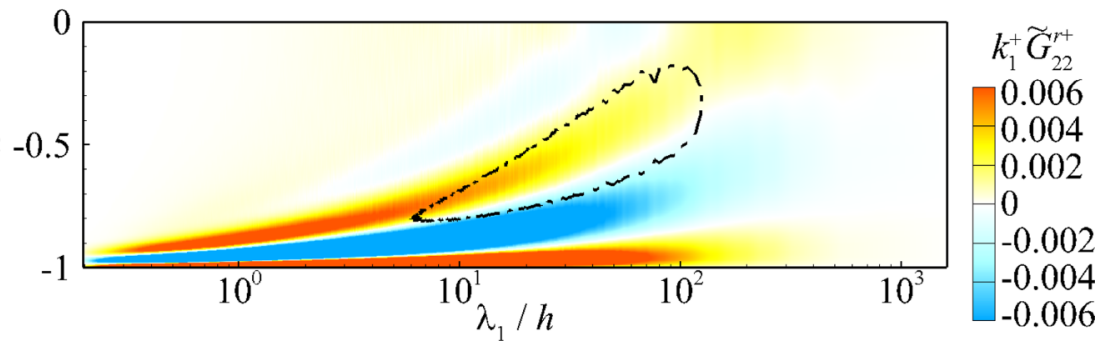

(e)
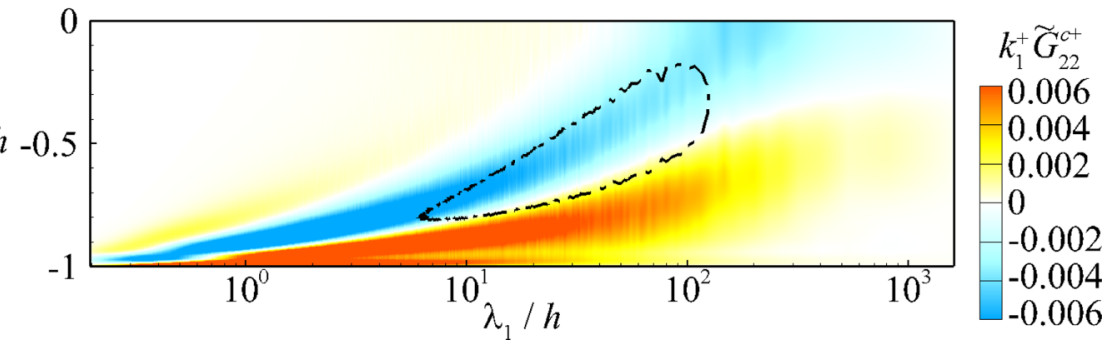

FIG. 9. Contours of key premultiplied budget terms in the transport equation of $\tilde{E}_{22}^{+}$on a $\lambda_{1}-x_{2}$ plane at $\operatorname{Ro}_{\tau}=150$. (a) Rotation-induced redistribution term $k_{1}^{+} \tilde{\Gamma}_{22}^{r+}$, (b) interscale transport term $k_{1}^{+} \tilde{T}_{22}^{s+}$, (c) wall-normal turbulent-diffusion term $k_{1}^{+} \tilde{T}_{22}^{p+}$, (d) rotation-induced pressure-diffusion term $k_{1}^{+} \tilde{G}_{22}^{r+}$, and (e) convection-induced pressure-diffusion term $k_{1}^{+} \tilde{G}_{22}^{c+}$. The dashed-dotted line represents the isopleth of $k_{1}^{+} \tilde{E}_{22}^{+}=0.85 \max \left(k_{1}^{+} \tilde{E}_{22}^{+}\right)$. The wave-number $\lambda_{1}$ is shown in a logarithmic coordinate. 

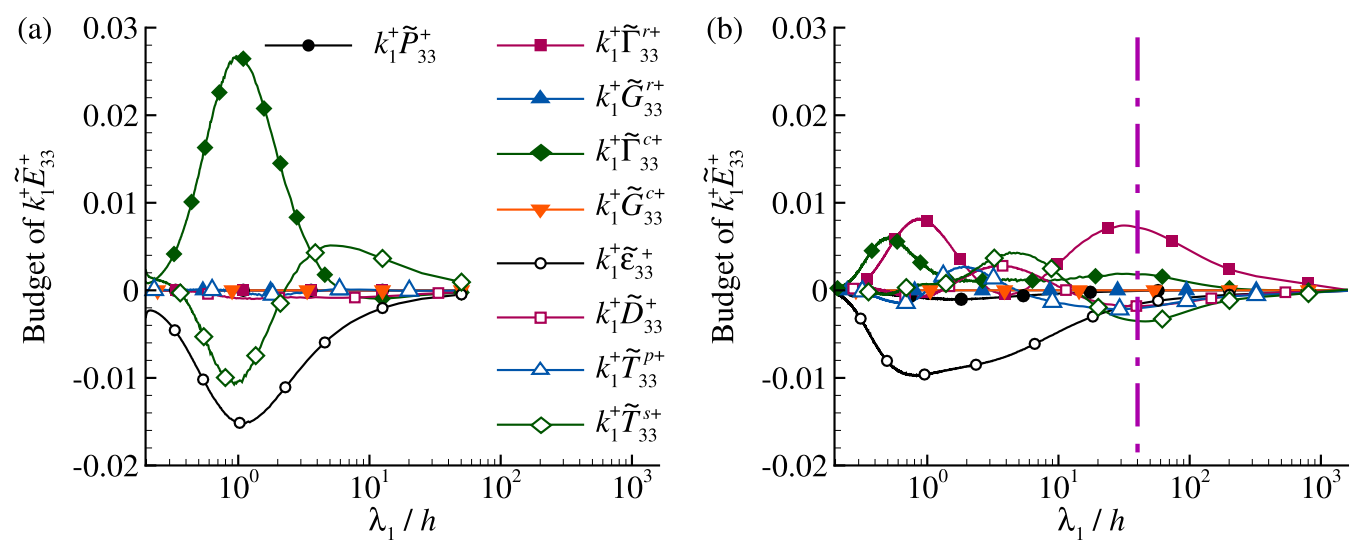

FIG. 10. Premultiplied budget terms in the transport equation of spectrum $\tilde{E}_{33}^{+}$at $x_{2} / h=-0.9$ for (a) nonrotating $\left(\mathrm{Ro}_{\tau}=0\right)$ and (b) streamwise-rotating $\left(\mathrm{Ro}_{\tau}=150\right)$ turbulent channel flows. The vertical dashed-dotted line demarcates the characteristic streamwise wavelength corresponding to the maximum value of $k_{1}^{+} \tilde{E}_{33}^{+}$[marked by the cross symbol in Fig. 3(c)]. The wave-number $\lambda_{1}$ is shown in a logarithmic coordinate.

values of $k_{1}^{+} \tilde{G}_{22}^{r+}$ [Fig. 9(d)], whereas the energy loss to the convection-induced pressure-diffusion term mainly feeds back to the near-wall region with positive values of $k_{1}^{+} \tilde{G}_{22}^{c+}$.

In summary of the budget analyses of $\tilde{E}_{22}^{+}$, the enhancement of $k_{1}^{+} \tilde{E}_{22}^{+}$at large wavelengths in the core region of the TGL vortices [i.e., feature (ii) of the energy spectra summarized in Sec. III] is induced by the combined effects of the rotation-induced redistribution, wall-normal turbulent diffusion, and interscale energy transfer. The rotation-induced redistribution process transfers energy from $\tilde{E}_{11}^{+}$to $\tilde{E}_{22}^{+}$at scales that are either larger or smaller than the characteristic streamwise length scales of the wall-normal motions induced by the TGL vortices. The TGL vortices feature a self-enhancement behavior through the wall-normal turbulent diffusion process that transports energy from the near-wall region to the core region of the TGL vortices around $x_{2} / h=-0.5$. The energy gained by $\tilde{E}_{22}^{+}$through the wall-normal turbulent diffusion process mainly concentrates at length scales that are smaller than the characteristic streamwise length scales of wall-normal motions induced by the TGL vortices. The energy at both smaller and larger scales is eventually gained by the TGL vortices through the interscale energy transport process.

At last, we examine the budget balance of the energy spectra of spanwise velocity fluctuations $\tilde{E}_{33}^{+}$. Figures 10 and 11 compare the premultiplied budget terms of $\tilde{E}_{33}^{+}$between the nonrotating and the streamwise-rotating channel flows at $x_{2}=-0.9$ and 0 , respectively. An interesting observation from these two figures is that the functions of the interscale transport term $\tilde{T}_{33}^{s+}$ are opposite between the nonrotating and the streamwise-rotating channel flows in both the near-wall region $\left(x_{2} / h=-0.9\right)$ and the central region $\left(x_{2} / h=0\right)$ of the channel. As shown in Fig. 10(a), in the near-wall region of the nonrotating channel flow, the value of $k_{1}^{+} \tilde{T}_{33}^{s+}$ is negative and positive at smaller wavelengths for $\lambda_{1} / h<2$ and larger wavelengths for $\lambda_{1} / h>3$, respectively, indicating inverse energy transport from small to large streamwise length scales in the near-wall region. By applying an averaging conditioned by a negative TKE production, Hamba [38] observed that the inverse energy transport is associated with vortex structures elongated in the streamwise direction. The spectral analyses also suggest that such an inverse energy transport in nonrotating channel flow is a direct consequence of vortex motions in the near-wall region [32,39,40]. The streamwise length scales of these vortices are, indeed, much smaller than those of the TGL vortices, and in the streamwise-rotating channel, these vortices are suppressed (Fig. 4). As a result, in the streamwiserotating channel flow, the inverse energy transport is absent in the near-wall region [Fig. 10(b)]. However, in the central region, the inverse energy transport is seen in the streamwise-rotating channel only [Fig. 11(b)]. 

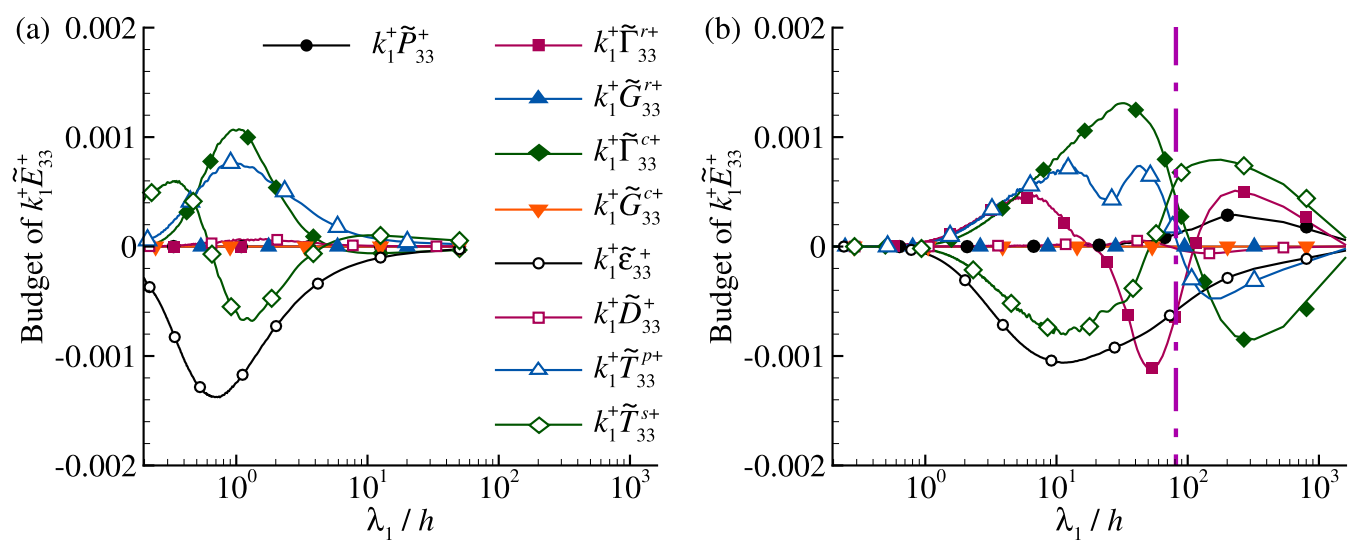

FIG. 11. Premultiplied budget terms in the transport equation of spectrum $\tilde{E}_{33}^{+}$at $x_{2} / h=0$ for (a) nonrotating $\left(\mathrm{Ro}_{\tau}=0\right)$ and (b) streamwise-rotating $\left(\mathrm{Ro}_{\tau}=150\right)$ turbulent channel flows. The vertical dashed-dotted line demarcates the characteristic wavelengths corresponding to the secondary peak of $k_{1}^{+} \tilde{E}_{33}^{+}$[marked by the triangle symbol in Fig. 3(c)]. The wave-number $\lambda_{1}$ is shown in a logarithmic coordinate.

To further investigate the vortex structures responsible for the inverse interscale energy transport in the center region of the streamwise rotating channel, we decompose the velocity fluctuations $u_{i}^{\prime}$ into a large-scale part $u_{i}^{L}$ and a small-scale part $u_{i}^{S}$ based on a cutoff filter at $\lambda_{1}=\lambda_{c}$ in the spectral space. Since we focus on the inverse interscale energy transport shown in Fig. 11(b), the cutoff wavelength is set to $\lambda_{c}=50$ at which $k_{1}^{+} T_{33}^{s+}=0$. Kawata and Alfredsson [9] adopted a similar decomposition based on the spanwise wavelength to analyze their experimental data of spanwiserotating channel flows. They noted that the interscale and wall-normal transports of Reynolds stresses are closely connected with the large-scale roll cells. By applying the decomposition of velocity fluctuations, the interscale transport term $T_{i j}^{s}$ can be further decomposed into the contributions from the large-scale motions $T_{i j}^{s, L}$, small-scale motions $T_{i j}^{s, L}$, and interactions between large- and small-scale motions $T_{i j}^{s, L S}$, defined respectively, as

$$
\begin{aligned}
& \tilde{T}_{i j}^{s, L}=\operatorname{Re}\left\{\frac{1}{2}\left(\frac{\partial \widehat{u_{i}^{L} u_{2}^{L} \widehat{u}_{j}^{L}}}{\partial x_{2}}+\frac{\partial \widehat{u_{j}^{L} u_{2}^{L} \widehat{u}_{i}^{L^{*}}}}{\partial x_{2}}\right)-i k_{1}\left(\widehat{\widehat{u}_{j}^{L} \widehat{u}_{i}^{u_{1}^{L}}{ }^{*}}-\overline{u_{i}^{L}} \widehat{u}_{j}^{u_{1}^{L}}\right)\right.
\end{aligned}
$$

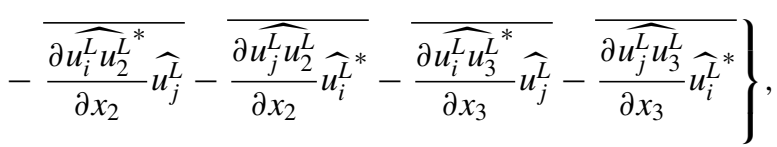

$$
\begin{aligned}
& \tilde{T}_{i j}^{S, S}=\operatorname{Re}\left\{\frac{1}{2}\left(\frac{\partial \widehat{u_{i}^{S u_{2}^{S}} \widehat{u}_{j}^{S}}}{\partial x_{2}}+\frac{\partial \widehat{u_{j}^{S} u_{2}^{S} \widehat{u}_{i}^{*}}}{\partial x_{2}}\right)-i k_{1}\left(\widehat{\widehat{u_{j}^{S}} \widehat{u_{i}^{S} u_{1}^{S^{*}}}}-\overline{\hat{u}_{i}^{*} \widehat{u}_{j}^{S} u_{1}^{S}}\right)\right. \\
& \left.-\widehat{\frac{\partial u_{i}^{S} u_{2}^{S^{*}}}{\partial x_{2}} \widehat{u_{j}^{S}}}-\overline{\frac{\partial u_{j}^{S} u_{2}^{S}}{\partial x_{2}} \widehat{u}_{i}^{*}}-\overline{\frac{\partial u_{i}^{S} u_{3}^{*}}{\partial x_{3}} \widehat{u_{j}^{S}}}-\widehat{\frac{\partial u_{j}^{S} u_{3}^{S}}{\partial x_{3}} \widehat{u}_{i}^{*}}\right\}, \\
& \tilde{T}_{i j}^{s, L S}=\tilde{T}_{i j}^{s}-\tilde{T}_{i j}^{s, L}-\tilde{T}_{i j}^{s, S} .
\end{aligned}
$$

Figure 12 compares the premultiplied interscale transport term $k_{1}^{+} T_{33}^{s+}$ and the three corresponding parts $k_{1}^{+} T_{33}^{s, L+}, k_{1}^{+} T_{33}^{s, S+}$, and $k_{1}^{+} T_{33}^{s, L S+}$. It is seen that the values of $k_{1}^{+} T_{33}^{s, L+}$ and $k_{1}^{+} T_{33}^{s, S+}$ are almost zero at $\lambda_{1}<\lambda_{c}$ and $\lambda_{1}>\lambda_{c}$, respectively. This indicates that either large-scale or small-scale 


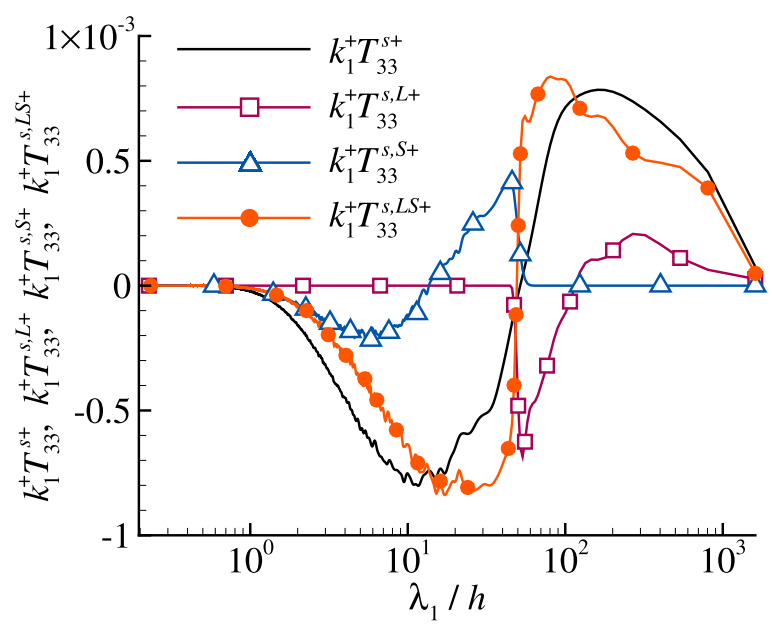

FIG. 12. Premultiplied interscale transport term $k_{1}^{+} T_{33}^{s+}$ and the contributions from the large-scale motions $k_{1}^{+} T_{33}^{s, L+}$, small-scale motions $k_{1}^{+} T_{33}^{s, S+}$, and interactions between large- and small-scale motions $k_{1}^{+} T_{33}^{s, L S+}$ at $x_{2} / h=0$ for streamwise rotating channel flow $\left(\mathrm{Ro}_{\tau}=150\right)$. The wave-number $\lambda_{1}$ is shown in a logarithmic coordinate.

motions are insufficient to induce inverse interscale energy transport. The magnitude of $k_{1}^{+} T_{33}^{s, L S+}$ is larger than that of either $k_{1}^{+} T_{33}^{s, L+}$ or $k_{1}^{+} T_{33}^{s, S+}$, indicating that the interactions between large-scale and small-scale motions are responsible for the inverse energy transport in $\tilde{E}_{33}$ at the center region of the channel [Fig. 10(b)].

From Figs. 10 and 11 , it is seen that besides the interscale transport term $\tilde{T}_{33}^{s+}$, the rotationinduced redistribution term $\tilde{\Gamma}_{33}^{r+}$, wall-normal turbulent-diffusion term $\tilde{T}_{33}^{p+}$, and convection-induced redistribution term $\tilde{\Gamma}_{33}^{c+}$ act as important sources in the streamwise-rotating channel flow at different $x_{2}$ locations and wavelengths $\lambda_{1} / h$. To develop a deeper understanding of their roles in sustaining the motion of the TGL vortices, the contours of their premultiplied values are displayed in Fig. 13. As shown in Fig. 13(a), in the near-wall region, the rotation-induced redistribution term $\tilde{\Gamma}_{33}^{r+}$ is the dominant source that provides energy to sustain the large-scale spanwise motions. The region with large magnitude of $k_{1}^{+} \tilde{\Gamma}_{33}^{r+}$ collocates with the region for $k_{1}^{+} \tilde{E}_{33}^{+} \geqslant 0.85 \max \left(k_{1}^{+} \tilde{E}_{33}^{+}\right)$, indicating that the energy redistribution process induced by the imposed system rotation is mainly responsible for the enhancement of $k_{1}^{+} \tilde{E}_{33}^{+}$at large wavelengths around the bottom edge of the TGL vortices [i.e., feature (iii) of the energy spectra summarized in Sec. III].

Around the top edge of the TGL vortices $\left(x_{2} / h=0\right)$, the large-scale spanwise motion induced by the TGL vortices is mainly sustained by the interscale transport term $\tilde{T}_{33}^{s+}$ [Fig. 13(b)], through which the TGL vortices gain energy from small-scale eddies. To track the origin of this part of energy at relatively small length scales for $\lambda_{1} / h<40$ (smaller than the characteristic length scale $\lambda_{1} / h \approx 90$ corresponding to the secondary peak of $k_{1}^{+} \tilde{E}_{33}^{+}$), it is seen from Figs. 13(c) and 13(d) that the wall-normal turbulent-diffusion term $\tilde{T}_{33}^{p+}$ and convection-induced redistribution term $\tilde{\Gamma}_{33}^{c+}$ are the dominant sources. The function of $\tilde{T}_{33}^{p+}$ is to transport energy from the core region of the TGL vortices (around $x_{2} / h=-0.5$ ) to the top edge of them (around $x_{2} / h=0$ ). The energy gain through the convection-induced redistribution term $\tilde{\Gamma}_{33}^{c+}$ for $\lambda_{1} / h<40$ around $x_{2} / h=0$ mainly originates from $\tilde{E}_{22}^{+}$through negatively valued $\tilde{\Gamma}_{22}^{c+}$ at the same ranges of $x_{2} / h$ and $\lambda_{1} / h$ (not shown in the figure), whereas Fig. 9(c) shows that it is also the wall-normal turbulent diffusion term $\tilde{T}_{22}^{p+}$ that transports energy from the near-wall region to the channel center for $\lambda_{1} / h<40$ in the budget of $\tilde{E}_{22}^{+}$. The above analyses indicate that the wall-normal turbulent diffusion and interscale transport processes are important for sustaining the spanwise motions of the TGL vortices at the channel 

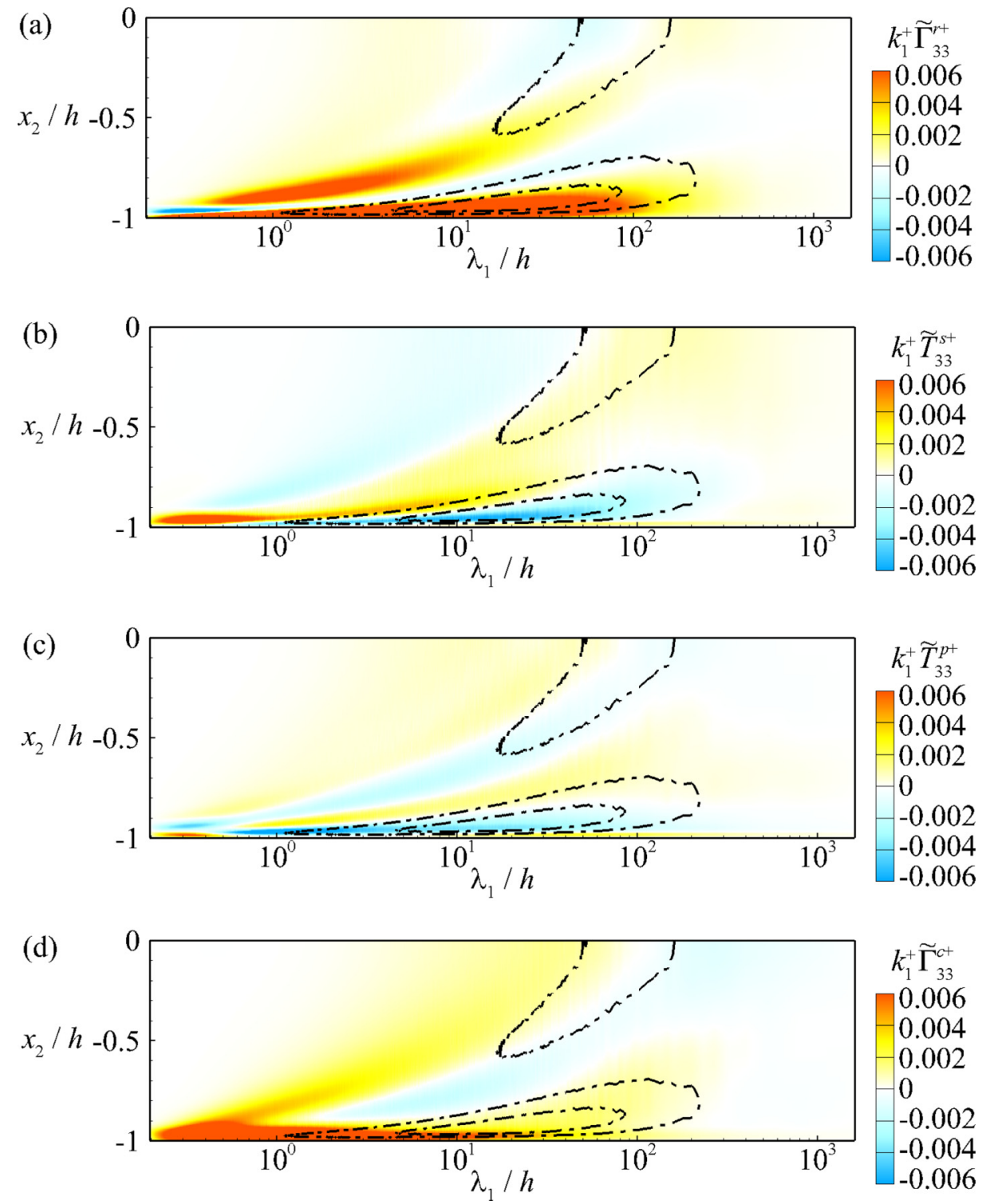

FIG. 13. Contours of key premultiplied budget terms in the transport equation of $\tilde{E}_{33}^{+}$on a $\lambda_{1}-x_{2}$ plane at $\mathrm{Ro}_{\tau}=150$. (a) Rotation-induced redistribution term $k_{1}^{+} \tilde{\Gamma}_{33}^{r+}$, (b) interscale transport term $k_{1}^{+} \tilde{T}_{33}^{s+}$, (c) wallnormal turbulent-diffusion term $k_{1}^{+} \tilde{T}_{33}^{p+}$, and (d) convection-induced redistribution term $k_{1}^{+} \tilde{\Gamma}_{33}^{c+}$. The dasheddotted lines represents the isopleths of $k_{1}^{+} \tilde{E}_{33}^{+}=0.85 \max \left(k_{1}^{+} \tilde{E}_{33}^{+}\right)$and $k_{1}^{+} \tilde{E}_{33}^{+}=0.5 \max \left(k_{1}^{+} \tilde{E}_{33}^{+}\right)$. The wavenumber $\lambda_{1}$ is shown in a logarithmic coordinate.

center that result in the enhancement of $k_{1}^{+} \tilde{E}_{33}^{+}$at large wavelengths around the top edge of the TGL vortices [i.e., feature (iv) of the energy spectra summarized in Sec. III].

\section{CONCLUSIONS}

To study the sustaining mechanism of the TGL vortices in the streamwise-rotating turbulent channel flows, we analyze the transport equation of energy spectra. Two new terms, namely, the 


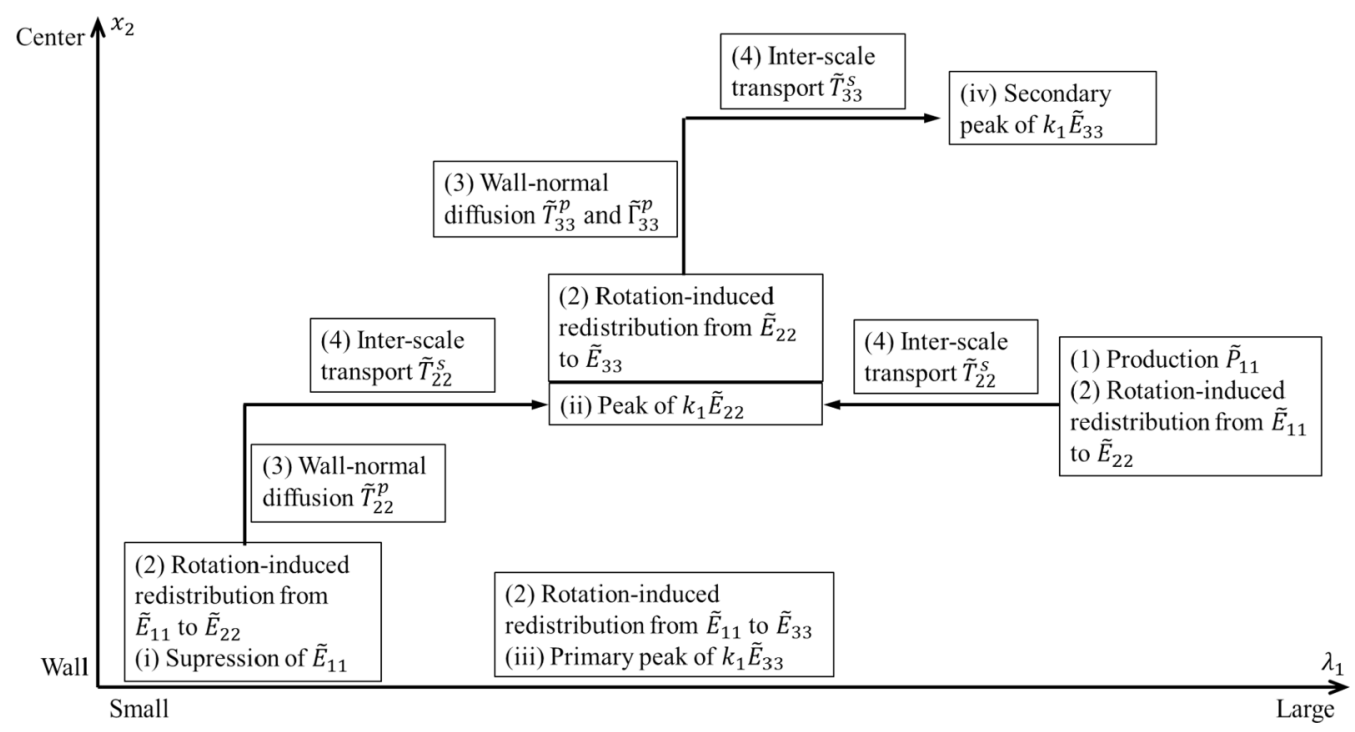

FIG. 14. Summary of the key features of the energy spectra $\tilde{E}_{i i}$ characterizing the motion of the TGL vortices in the streamwise-rotating channel flow and the dominant energy sustaining processes. The horizontal and vertical axes represent the streamwise length scale and wall-normal location, respectively.

rotation-induced redistribution term and rotation-induced pressure-diffusion term, are defined to investigate the effect of the imposed streamwise system rotation on the energy transport process.

Through the budget balance analyses of the energy spectra, it is discovered that there are four crucial physical processes that sustain the motion of large-scale the TGL vortices. Figure 14 summarizes these four energy sustaining processes of the TGL vortices. The four key features of the energy spectra $\tilde{E}_{i i}$ characterizing the motion of the TGL vortices summarized in Sec. III are also listed in the figure. As shown, the first process is the energy production at large length scales. Through this process, the large-scale TGL vortices are able to gain energy from the mean flow once they are induced in the channel. The second process is the additional energy redistribution from the streamwise velocity component to the wall-normal and spanwise components. In the nonrotating channel flow, the convection-induced pressure fluctuation plays an important role in the energy redistribution, but in the streamwise-rotating channel flow, its role is replaced by the rotation-induced pressure fluctuation. The magnitude of the rotation-induced redistribution term in the streamwise-rotating channel flow is larger than that of the convection-induced redistribution term in the nonrotating channel flow, indicating an enhancing effect of the streamwise system rotation on the wall-normal and spanwise velocity fluctuations to sustain the vortex motion in the cross-stream plane. The enhanced wall-normal diffusion is the third important process, which transports additional energy from the near-wall region to the channel center to sustain the motion of the TGL vortices. The last key process is the inverse interscale energy transport in the vertical and spanwise velocity fluctuations, through which the large-scale TGL vortices absorb energy from small-scale eddies.

\section{ACKNOWLEDGMENTS}

This research was supported by NSFC Basic Science Center Program for "Multiscale Problems in Nonlinear Mechanics" (Grant No. 11988102). Z.Y. would like to thank the LiXing research funding of the Institute of Mechanics, Chinese Academy of Sciences. Research funding from the Natural Sciences and Engineering Research Council (NSERC) of Canada to B.-C.W. is gratefully 
acknowledged. The National Supercomputer Center in Tianjin is acknowledged for access to supercomputing and storage facilities.

\section{APPENDIX: TRANSPORT EQUATION OF VELOCITY-SPECTRUM TENSOR}

The transport equation of the energy spectra $\tilde{E}_{i j}$ in a nonrotating channel flow has been given by Lumley [29], Mizuno [30], and Lee and Moser [32]. Here, we derive the equation in a streamwiserotating channel flow. Detailed analysis of the budget balance of $\tilde{E}_{i j}$ is presented in Sec. IV. The derivation starts with the following governing equation of instantaneous velocity fluctuations:

$$
\frac{\partial u_{i}^{\prime}}{\partial t}=-\left\langle u_{k}\right\rangle \frac{\partial u_{i}^{\prime}}{\partial x_{k}}-u_{k}^{\prime} \frac{\partial\left\langle u_{i}\right\rangle}{\partial x_{k}}-\frac{\partial\left(u_{i}^{\prime} u_{k}^{\prime}\right)}{\partial x_{k}}+\frac{\partial\left\langle u_{i}^{\prime} u_{k}^{\prime}\right\rangle}{\partial x_{k}}-\frac{\delta_{i k}}{\rho} \frac{\partial p^{\prime}}{\partial x_{k}}+v \frac{\partial^{2} u_{i}^{\prime}}{\partial x_{k} \partial x_{k}}-2 \epsilon_{i 1 k} \Omega u_{k}^{\prime} .
$$

Applying the Fourier transform (3) to the above equation yields

$$
\begin{aligned}
\frac{\partial \widehat{u_{i}^{\prime}}}{\partial t}= & -i k_{1}\left\langle u_{1}\right\rangle \widehat{u_{i}^{\prime}}-\left\langle u_{3}\right\rangle \frac{\partial \widehat{u_{i}^{\prime}}}{\partial x_{3}}-\widehat{u_{k}^{\prime}} \frac{\partial\left\langle u_{i}\right\rangle}{\partial x_{k}}-i k_{1} \widehat{u_{i}^{\prime} u_{1}^{\prime}}-\frac{\partial \widehat{u_{i}^{\prime} u_{2}^{\prime}}}{\partial x_{2}}-\frac{\partial \widehat{u_{i}^{\prime} u_{3}^{\prime}}}{\partial x_{3}} \\
& -\frac{1}{\rho} i k_{1} \widehat{p^{\prime}} \delta_{i 1}-\frac{1}{\rho} \frac{\partial \widehat{p^{\prime}}}{\partial x_{2}} \delta_{i 2}-\frac{1}{\rho} \frac{\partial \widehat{p}^{\prime}}{\partial x_{3}} \delta_{i 3}-v k_{1}^{2} \widehat{u_{i}^{\prime}}+v \frac{\partial^{2} \widehat{u_{i}^{\prime}}}{\partial x_{2}^{2}}+v \frac{\partial^{2} \widehat{u_{i}^{\prime}}}{\partial x_{3}^{2}}-2 \epsilon_{i 1 k} \Omega \widehat{u_{k}^{\prime}} .
\end{aligned}
$$

Note that the fourth term on the right-hand side of Eq. (A1) is independent of $x_{1}$ in the physical space. As a result, its Fourier transform is nontrivial only at $k_{1}=0$. Because the value of $\widehat{u_{i}^{\prime}}$ is zero at $k_{1}=0$, the Fourier transform of the fourth term on the right-hand side of Eq. (A1) does not show in Eq. (A2). Taking the conjugate of Eq. (A2) yields the governing equation of $\widehat{u_{i}^{\prime}}$, viz.,

$$
\begin{aligned}
\frac{\partial \widehat{u}_{i}^{\prime *}}{\partial t}= & i k_{1}\left\langle u_{1}\right\rangle{\widehat{u_{i}^{\prime}}}^{*}-\left\langle u_{3}\right\rangle \frac{\partial{\widehat{u_{i}}}_{i}^{*}}{\partial x_{3}}-{\widehat{u_{k}^{\prime}}}^{*} \frac{\partial\left\langle u_{i}\right\rangle}{\partial x_{k}}+i \widehat{k}_{1}{\widehat{u_{i}^{\prime} u_{1}^{\prime}}}^{*}-\frac{\partial \widehat{u_{i}^{\prime} u_{2}^{*}}}{\partial x_{2}}-\frac{\partial \widehat{u_{i}^{\prime} u_{3}^{\prime}}}{\partial x_{3}} \\
& +\frac{1}{\rho} i k_{1}{\widehat{p^{\prime}}}^{*} \delta_{i 1}-\frac{1}{\rho} \frac{\partial{\widehat{p^{\prime}}}^{*}}{\partial x_{2}} \delta_{i 2}-\frac{1}{\rho} \frac{\partial{\widehat{p^{\prime}}}^{*}}{\partial x_{3}} \delta_{i 3}-v \widehat{k}_{1}^{2}{\widehat{u_{i}^{\prime}}}^{*}+v \frac{\partial^{2}{\widehat{u_{i}^{\prime}}}^{*}}{\partial x_{2}^{2}}+v \frac{\partial^{2}{\widehat{u_{i}^{\prime}}}^{*}}{\partial x_{3}^{2}}-2 \epsilon_{i 1 k} \Omega{\widehat{u_{k}^{\prime}}}^{*} .
\end{aligned}
$$

Replacing subscript $i$ with $j$ in Eq. (A2) results in the governing equation of $\widehat{u_{j}^{\prime}}$ as

$$
\begin{aligned}
\frac{\partial \widehat{u_{j}^{\prime}}}{\partial t}= & -i k_{1}\left\langle u_{1}\right\rangle \widehat{u_{j}^{\prime}}-\left\langle u_{3}\right\rangle \frac{\partial \widehat{u_{j}^{\prime}}}{\partial x_{3}}-\widehat{u_{k}^{\prime}} \frac{\partial\left\langle u_{j}\right\rangle}{\partial x_{k}}-i k_{1} \widehat{u_{j}^{\prime} u_{1}^{\prime}}-\frac{\partial \widehat{u_{j}^{\prime} u_{2}^{\prime}}}{\partial x_{2}}-\frac{\partial \widehat{u_{j}^{\prime} u_{3}^{\prime}}}{\partial x_{3}} \\
& -\frac{1}{\rho} i k_{1} \widehat{p^{\prime}} \delta_{j 1}-\frac{1}{\rho} \frac{\partial \widehat{p}^{\prime}}{\partial x_{2}} \delta_{j 2}-\frac{1}{\rho} \frac{\partial \widehat{p}^{\prime}}{\partial x_{3}} \delta_{j 3}-v k_{1}^{2} \widehat{u_{j}^{\prime}}+v \frac{\partial^{2} \widehat{u_{j}^{\prime}}}{\partial x_{2}^{2}}+v \frac{\partial^{2} \widehat{u_{j}^{\prime}}}{\partial x_{3}^{2}}-2 \epsilon_{j 1 k} \Omega \widehat{u_{k}^{\prime}} .
\end{aligned}
$$

Multiplying Eqs. (A3) and (A4) with $\widehat{u_{j}^{\prime}}$ and ${\widehat{u_{i}^{\prime}}}^{*}$, respectively, and taking the summation of the two resultant equations leads to

$$
\begin{aligned}
& \frac{\partial\left(\widehat{u_{i}^{\prime}} \cdot \widehat{u_{j}^{\prime}}\right)}{\partial t}=-\left\langle u_{3}\right\rangle \frac{\partial\left(\widehat{u_{i}^{\prime}} * \widehat{u_{j}^{\prime}}\right)}{\partial x_{3}}-\widehat{u_{j}^{\prime}} \widehat{u_{k}^{\prime}} * \frac{\partial\left\langle u_{i}\right\rangle}{\partial x_{k}}-\widehat{u_{i}^{\prime}} * \widehat{u_{k}^{\prime}} \frac{\partial\left\langle u_{j}\right\rangle}{\partial x_{k}}-i k_{1}\left(\widehat{u_{j}^{\prime}} \widehat{u_{i}^{\prime} u_{1}^{\prime}} * \widehat{u_{i}^{*}} * \widehat{u_{j}^{\prime} u_{1}^{\prime}}\right)-\frac{\partial \widehat{u_{i}^{\prime} u_{2}^{\prime}}}{\partial x_{2}} \widehat{u_{j}^{\prime}} \\
& -\frac{\partial \widehat{u_{j}^{\prime} u_{2}^{\prime}}}{\partial x_{2}} \widehat{u}_{i}^{\prime *}-\frac{\partial \widehat{u_{i}^{\prime} u_{3}^{\prime}}}{\partial x_{3}} \widehat{u_{j}^{\prime}}-\frac{\partial \widehat{u_{j}^{\prime} u_{3}^{\prime}}}{\partial x_{3}} \widehat{u}_{i}^{\prime *}-\frac{1}{\rho} i k_{1}\left(\widehat{p}^{\prime} * \widehat{u_{j}^{\prime}} \delta_{i 1}-\widehat{p^{\prime} \widehat{u}_{i}^{\prime}} \delta_{j 1}\right) \\
& -\frac{1}{\rho}\left(\frac{\partial \widehat{p}^{*}}{\partial x_{2}} \widehat{u_{j}^{\prime}} \delta_{i 2}+\frac{\partial \widehat{p^{\prime}}}{\partial x_{2}}{\widehat{u_{i}^{\prime}}}^{*} \delta_{j 2}\right)-\frac{1}{\rho}\left(\frac{\partial \widehat{p}^{*}}{\partial x_{3}} \widehat{u_{j}^{\prime}} \delta_{i 3}+\frac{\partial \widehat{p}^{\prime}}{\partial x_{3}} \widehat{u}_{i}^{\prime *} \delta_{j 3}\right)-2 \nu k_{1}^{2} \widehat{u_{i}^{\prime}} * \widehat{u_{j}^{\prime}}
\end{aligned}
$$

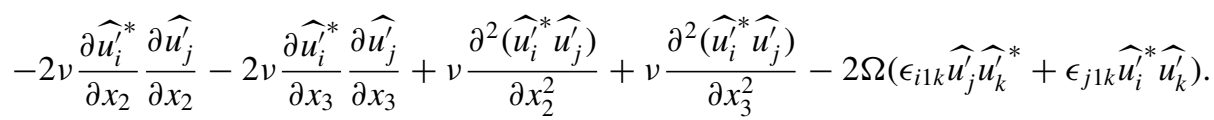


Decomposing the pressure term into a redistribution part and a wall-normal diffusion part and further applying the decomposition of pressure fluctuations $p^{\prime}$ into the rotation-induced part $p_{r}^{\prime}$ and convection-induced part $p_{c}^{\prime}$, Eq. (A5) is rewritten as

$$
\begin{aligned}
& \frac{\partial\left({\widehat{u_{i}^{\prime}}}^{*} \widehat{u_{j}^{\prime}}\right)}{\partial t}=-\widehat{u_{j}^{\prime}}{\widehat{u_{k}^{\prime}}}^{*} \frac{\partial\left\langle u_{i}\right\rangle}{\partial x_{k}}-\widehat{u_{i}^{\prime}} * \widehat{u_{k}^{\prime}} \frac{\partial\left\langle u_{j}\right\rangle}{\partial x_{k}}-2 \Omega\left(\epsilon_{i 1 k} \widehat{u_{j}^{\prime}}{\widehat{u_{k}^{\prime}}}^{*}+\epsilon_{j 1 k} \widehat{u_{i}^{\prime}} * \widehat{u_{k}^{\prime}}\right)-\frac{1}{\rho} i k_{1}\left({\widehat{p_{r}^{\prime}}}^{*} \widehat{u_{j}^{\prime}} \delta_{i 1}-\widehat{p_{r}^{\prime}}{\widehat{u_{i}^{\prime}}}^{*} \delta_{j 1}\right)
\end{aligned}
$$

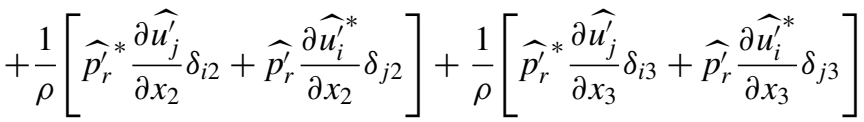

$$
\begin{aligned}
& -\frac{1}{\rho}\left[\frac{\partial\left({\widehat{p_{r}^{\prime}}}^{*}{\widehat{u_{j}^{\prime}}}^{\prime}\right)}{\partial x_{2}} \delta_{i 2}+\frac{\partial\left(\widehat{p_{r}^{\prime}}{\widehat{u_{i}^{\prime}}}^{*}\right)}{\partial x_{2}} \delta_{j 2}\right]-\frac{1}{\rho}\left[\frac{\partial\left({\widehat{p_{r}^{\prime}}}^{*}{\widehat{u_{j}^{\prime}}}^{\prime}\right)}{\partial x_{3}} \delta_{i 3}+\frac{\partial\left(\widehat{p_{r}^{\prime}}{\widehat{u_{i}^{\prime}}}^{*}\right)}{\partial x_{3}} \delta_{j 3}\right]
\end{aligned}
$$

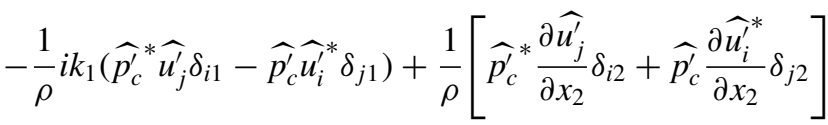

$$
\begin{aligned}
& +\frac{1}{\rho}\left[{\widehat{p_{c}^{\prime}}}_{c}^{*} \frac{\partial{\widehat{u_{j}^{\prime}}}_{\partial x_{3}}}{\partial i 3}+\widehat{p_{c}^{\prime}} \frac{\partial{\widehat{u_{i}^{\prime}}}^{*}}{\partial x_{3}} \delta_{j 3}\right]-\frac{1}{\rho}\left[\frac{\partial\left({\widehat{p_{c}^{\prime}}}^{*}{\widehat{u_{j}^{\prime}}}^{2}\right)}{\partial x_{2}} \delta_{i 2}+\frac{\partial\left(\widehat{p_{c}^{\prime}}{\widehat{u_{i}^{\prime}}}^{*}\right)}{\partial x_{2}} \delta_{j 2}\right] \\
& -\frac{1}{\rho}\left[\frac{\partial\left(\widehat{p}_{c}^{\prime} * \widehat{u}_{j}^{\prime}\right)}{\partial x_{3}} \delta_{i 3}+\frac{\partial\left(\widehat{p_{c}^{\prime}}{\widehat{u_{i}^{\prime}}}^{*}\right)}{\partial x_{3}} \delta_{j 3}\right]-2 v k_{1}^{2}{\widehat{u_{i}^{\prime}}}^{*} \widehat{u_{j}^{\prime}}-2 v \frac{\partial \widehat{u}_{i}^{*}}{\partial x_{2}} \frac{\partial \widehat{u}_{j}^{\prime}}{\partial x_{2}} \\
& -2 v \frac{\partial{\widehat{u_{i}^{\prime}}}^{*}}{\partial x_{3}} \frac{\partial \widehat{u_{j}^{\prime}}}{\partial x_{3}}+v \frac{\partial^{2}\left(\widehat{u_{i}^{\prime}} * \widehat{u_{j}^{\prime}}\right)}{\partial x_{2}^{2}}+v \frac{\partial^{2}\left(\widehat{u_{i}^{\prime}} * \widehat{u_{j}^{\prime}}\right)}{\partial x_{3}^{2}}-\frac{1}{2}\left[\frac{\partial\left(\widehat{u_{i}^{\prime} u_{2}^{\prime}} * \widehat{u_{j}^{\prime}}\right)}{\partial x_{2}}+\frac{\partial\left(\widehat{u_{j}^{\prime} u_{2}^{\prime}}{\widehat{u_{i}}}^{*}\right)}{\partial x_{2}}\right] \\
& +\frac{1}{2}\left[\frac{\partial\left(\widehat{u_{i}^{\prime} u_{2}^{\prime}} * \widehat{u_{j}^{\prime}}\right)}{\partial x_{2}}+\frac{\partial\left(\widehat{u_{j}^{\prime} u_{2}^{\prime}}{\widehat{u_{i}^{\prime}}}^{*}\right)}{\partial x_{2}}\right]-i k_{1}\left(\widehat{u_{j}^{\prime}} \widehat{u_{i}^{\prime} u_{1}^{\prime}} *{\widehat{u_{i}^{\prime}}}^{*} \widehat{u_{j}^{\prime} u_{1}^{\prime}}\right) \\
& -\frac{\partial \widehat{u_{i}^{\prime} u_{2}^{\prime}}}{\partial x_{2}}{\widehat{u_{j}^{\prime}}}^{*}-\frac{\partial \widehat{u_{j}^{\prime} u_{2}^{\prime}}}{\partial x_{2}}{\widehat{u_{i}^{\prime}}}^{*}-\frac{\partial \widehat{u_{i}^{\prime} u_{3}^{\prime}}}{\partial x_{3}} \widehat{u_{j}^{\prime}}-\frac{\partial \widehat{u_{j}^{\prime} u_{3}^{\prime}}}{\partial x_{3}}{\widehat{u_{i}^{\prime}}}^{*}-\left\langle u_{3}\right\rangle \frac{\partial\left(\widehat{u_{i}^{\prime}} * \widehat{u_{j}^{\prime}}\right)}{\partial x_{3}} \text {. }
\end{aligned}
$$

Finally, performing time and spanwise averaging over Eq. (A6) and taking the real part of the resultant equation results in the transport equation (4) of the velocity-spectrum tensor $\tilde{E}_{i j}$.

[1] D. P. Wall and M. Nagata, Nonlinear secondary flow through a rotating channel, J. Fluid Mech. 564, 25 (2006).

[2] S. Masuda, S. Fukuda, and M. Nagata, Instabilities of plane Poiseuille flow with a streamwise system rotation, J. Fluid Mech. 603, 189 (2008).

[3] R. Kristoffersen and H. I. Andersson, Direct simulations of low-Reynolds-number turbulent flow in a rotating channel, J. Fluid Mech. 256, 163 (1993).

[4] O. Grundestam, S. Wallin, and A. V. Johansson, Direct numerical simulations of rotating turbulent channel flow, J. Fluid Mech. 598, 177 (2008).

[5] N.-S. Liu and X.-Y. Lu, A numerical investigation of turbulent flows in a spanwise rotating channel, Comp. Fluids 36, 282 (2007).

[6] Y.-J. Dai, W.-X. Huang, and C.-X. Xu, Effects of Taylor-Görtler vortices on turbulent flows in a spanwiserotating channel, Phys. Fluids 28, 115104 (2016).

[7] Z. Xia, Y. Shi, Q. Cai, M. Wan, and S. Chen, Multiple states in turbulent plane Couette flow with spanwise rotation, J. Fluid Mech. 837, 477 (2018). 
[8] Y. Huang, Z. Xia, M. Wan, Y. Shi, and S. Chen, Hysteresis behavior in spanwise rotating plane Couette flow with varying rotation rates, Phys. Rev. Fluids 4, 052401(R) (2019).

[9] T. Kawata and P. H. Alfredsson, Scale interactions in turbulent rotating planar Couette flow: Insight through the Reynolds stress transport, J. Fluid Mech. 879, 255 (2019).

[10] P. Bradshaw, The analogy between streamline curvature and buoyancy in turbulent shear flow, J. Fluid Mech. 36, 177 (1969).

[11] J. E. Hart, Instability and secondary motion in a rotating channel flow, J. Fluid Mech. 45, 341 (1971).

[12] J. P. Johnston, R. M. Halleen, and D. K. Lezius, Effects of spanwise rotation on the structure of twodimensional fully developed turbulent channel flow, J. Fluid Mech. 56, 533 (1972).

[13] D. K. Lezius and J. P. Johnston, Roll-cell instabilities in rotating laminar and turbulent channel flows, J. Fluid Mech. 77, 153 (1976).

[14] D. J. Tritton and P. A. Davies, Instabilities in geophysical fluid dynamics, in Hydrodynamic Instabilities and the Transition to Turbulence, 2nd ed., edited by H. L. Swinney and J. P. Gollub (Springer, Berlin, 1985), pp. 229-270.

[15] Z. Yang and B.-C. Wang, Capturing Taylor-Görtler vortices in a streamwise-rotating channel at very high rotation numbers, J. Fluid Mech. 838, 658 (2018).

[16] Z. Yang, B.-Q. Deng, B.-C. Wang, and L. Shen, On the mechanism of the formation of Taylor-Görtler-like vortices in fast streamwise-rotating channel flows, Phys. Fluids 30, 091701 (2018).

[17] Y.-J. Dai, W.-X. Huang, and C.-X. Xu, Coherent structures in streamwise rotating channel flow, Phys. Fluids 31, 021204 (2019).

[18] T. Weller and M. Oberlack, DNS of a turbulent channel flow with streamwise rotation - investigation on the cross flow phenomena, in Direct and Large-Eddy Simulation VI, edited by E. Lamballais, R. Friedrich, B. J. Geurts, and O. Métais (Springer, Berlin, 2006), pp. 241-248.

[19] T. Weller and M. Oberlack, DNS of a turbulent channel flow with streamwise rotation-study of the reverse effect of the cross flow, Proc. Appl. Math. Mech. 6, 553 (2006).

[20] N. N. Mansour, J. Kim, and P. Moin, Reynolds-stress and dissipation-rate budgets in a turbulent channel flow, J. Fluid Mech. 194, 15 (1988).

[21] R. A. Antonia and J. Kim, Low-Reynods-number effects on near-wall turbulence, J. Fluid Mech. 276, 61 (1994).

[22] R. D. Moser, J. Kim, and N. N. Mansour, Direct numerical simulation of turbulent channel flow up to $\operatorname{Re}_{\tau}=590$, Phys. Fluids 11, 943 (1999).

[23] S. Hoyas and J. Jiménez, Reynolds number effects on the Reynolds-stress budgets in turbulent channels, Phys. Fluids 20, 101511 (2008).

[24] J. Komminaho and M. Skote, Reynolds stress budgets in Couette and boundary layer flows, Flow, Turbul. Combust. 68, 167 (2002).

[25] V. Avsarkisov, S. Hoyas, M. Oberlack, and J. P. García-Galache, Turbulent plane Couette flow at moderately high Reynolds number, J. Fluid Mech. 751, R1 (2014).

[26] P. R. Spalart, Direct simulation of a turbulent boundary layer up to $R_{\theta}=1410$, J. Fluid Mech. 187, 61 (1988).

[27] E. Lamballais, M. Lesieur, and O. Métais, Effects of spanwise rotation on the vorticity stretching in transitional and turbulent channel flow, Int. J. Heat Fluid Flow 17, 324 (1995).

[28] Z. Yang, B.-Q. Deng, and B.-C. Wang, Effect of fast rotation on pressure fluctuations and TaylorGörtler-like vortices in channel flows, Eleventh International Symposium on Turbulence and Shear Flow Phenomena (TSFP11) (University of Southampton, Southampton, UK, 2019).

[29] J. L. Lumley, Spectral energy budget in wall turbulence, Phys. Fluids 7, 190 (1964).

[30] Y. Mizuno, Spectra of energy transport in turbulent channel flows for moderate Reynolds numbers, J. Fluid Mech. 805, 171 (2016).

[31] M. Lee and R. D. Moser, Spectral analysis on Reynolds stress transport equation in high Re wallbounded turbulence. Ninth International Symposium on Turbulence and Shear Flow Phenomena (TSFP9) (University of Melbourne, Melbourne, Australia, 2015).

[32] M. Lee and R. D. Moser, Spectral analysis of the budget equation in turbulent channel flows at high Reynolds number, J. Fluid Mech. 860, 886 (2019). 
[33] T. Kawata and P. H. Alfredsson, Inverse Interscale Transport of the Reynolds Shear Stress in Plane Couette Turbulence, Phys. Rev. Lett. 120, 244501 (2018).

[34] T. Kawata and T. Tsukahara, Numerical invesitigation on scale-by-scale Reynolds stress transport in plane Couette turbulence, Ninth International Symposium on Turbulence and Shear Flow Phenomena (TSFP11) (University of Southampton, Southampton, UK, 2019).

[35] B.-Q. Deng and C.-X. Xu, Influence of active control on STG-based generation of streamwise vortices in near-wall turbulence, J. Fluid Mech. 710, 234 (2012).

[36] Z. Yang, G. Cui, C.-X. Xu, and Z.-S. Zhang, Large eddy simulation of rotating turbulent channel flow with a new dynamic global-coefficient nonlinear subgrid stress model, J. Turbul. 13, 48 (2012).

[37] Z. Yang, G. Cui, Z.-S. Zhang, and C.-X. Xu, A modified nonlinear sub-grid scale model for large eddy simulation with application to rotating turbulent channel flows, Phys. Fluids 24, 075113 (2012).

[38] F. Hamba, Inverse energy cascade and vortical structure in the near-wall region of turbulent channel flow, Phys. Rev. Fluids 4, 114609 (2019).

[39] A. Cimarelli, E. De Angelis, and C. M. Casciola, Paths of energy in turbulent channel flows, J. Fluid Mech. 715, 436 (2013).

[40] A. Cimarelli, E. De Angelis, J. Jiménez, and C. M. Casciola, Cascades and wall-normal fluxes in turbulent channel flows, J. Fluid Mech. 796, 417 (2016). 